\title{
Comparação entre diferentes abordagens do problema de fluxo de potência ótimo utilizando o método de pontos interiores
}

\author{
Aline Maria de Lima
}

Orientador: Prof. Dr. Aurelio Ribeiro Leite de Oliveira

Dissertação apresentada ao Instituto de Ciências Matemáticas e de Computação - ICMC-USP, como parte dos requisitos para obtenção do título de Mestre em Ciências - Área: Ciências da Computação e Matemática Computacional.

USP - São Carlos - SP

Agosto/2004 
Aos meus pais, irmã e avó. 


\section{Agradecimentos}

À Deus, que com Seu amor, me guiou e me fortaleceu em mais esta etapa de minha vida.

Ao meu orientador Aurelio pela orientação e pela confiança despositada em mim para o desenvolvimento deste trabalho e de outros que virão. Agradeço a atenção dispensada em todos os momentos, e as explicações e sugestões dadas não só para o melhor desenvolvimento do projeto, mas também para meu crescimento de forma geral.

À FAPESP - Fundação de Amparo e Apoio à Pesquisa do Estado de São Paulo, pela credibilidade e apoio financeiro.

Aos meus pais, Ero e Milton, pelo amor e apoio incondicionais em todos os momentos da minha vida. Agradeço por me possibilitarem chegar até aqui, doando-se por inteiro para que eu pudesse cumprir mais esta etapa. Agradeço a Deus todos os dias por vocês serem meus pais, e nunca poderei retribuir tudo o que fazem por mim. Amo vocês.

À minha querida avó Marcolina pelo imenso carinho e amor dedicados a mim. Obrigada pelas orações e pelo apoio em todos os momentos. Obrigada por ficar feliz com as minhas alegrias e pelo conforto dado em minhas tristezas.

À minha irmã Paty, por ser muito mais que uma irmã. Fí, você é uma extensão de mim, é uma pessoa que eu sei que estará sempre comigo, sempre do meu lado, em tudo que eu fizer. Obrigada pelo incentivo e apoio durante esta caminhada. Irmã, "trust I seek, and I find in you, everyday for us something new, open mind for a different view, and nothing else matters".

Ao Christian, pelo amor, carinho, amizade, compreensão e apoio dados a mim 
todos estes anos. Obrigada por estar sempre ao meu lado, me ajudando em tudo que eu preciso. Você é muito importante para mim, e sei que estará ao meu lado, sendo meu anjo salvador pelo resto da minha vida.

Às minhas amigas de São Carlos, por estarem sempre dispostas a me ajudar e pelo apoio, carinho e amizade.

Aos professores e funcionários do ICMC-USP que, direta ou indiretamente, contribuíram com este trabalho.

E finalmente a todos que colaboraram indiretamente para a realização deste trabalho. 


\section{Resumo}

Neste trabalho, é apresentado o problema de fluxo de potência ótimo DC utilizando os modelos de fluxos em rede e o princípio do mínimo esforço. É apresentada também uma revisão dos métodos de pontos interiores e o mesmo é desenvolvido para um problema geral de programação linear. Em seguida, desenvolvemos os métodos de pontos interiores primal-dual e preditor-corretor para os modelos de fluxo de potência específicos estudados. Resultados numéricos de uma implementação em MATLAB são apresentados para sistemas testes do IEEE e sistemas reais, comparando o desempenho obtido pelas duas formulações. O método de pontos interiores se mostra bastante robusto convergindo rapidamente para todos casos testados. O modelo usando o princípio do mínimo esforço converge em menos iterações, mas a modelagem por fluxos em rede se mostra mais rápida, uma vez que suas iterações são mais baratas. 


\section{Abstract}

In this work, the optimal DC power flow problem using the network flow models, and the minimum effort principle are presented. Also is presented a review of the interior point methods and this method is developed for a generic linear programming problem. We developed the primal-dual and predictor-corrector versions of the interior point methods for the specific power flow models studied. Numerical results of a MATLAB implementation are presented for IEEE test systems and for real systems, confronting the performances achieved for both approaches. The interior point methods shows to be robust, achieving fast convergence in all instances tested. The minimum effort principle based model converges in fewer iterations, but the network flow model shows to be faster, since its iterations are cheaper. 


\section{Conteúdo}

1 Introdução 1

2 O Problema de Fluxo de Potência Ótimo DC 3

2.1 Sistemas de Potência . . . . . . . . . . . . . . . . . . . . . 3

2.2 Modelagem Matemática do Problema de Fluxo de Potência Ótimo . . . . . 4

2.3 O Princípio do Mínimo Esforço . . . . . . . . . . . . . . . . . . . . . . 8

3 Métodos de Pontos Interiores 12

3.1 Conceitos Iniciais sobre Pontos Interiores . . . . . . . . . . . . . . 12

3.2 Usando o Método de Newton para Construir Métodos de Pontos Interiores 13

3.3 Método de Pontos Interiores Primal-Dual . . . . . . . . . . . . . . . . 17

3.4 Método de Pontos Interiores Primal-Dual com Variáveis Canalizadas . . . 18

3.4.1 Formulações Primal e Dual . . . . . . . . . . . . . . . . . . . . . 18

3.4.2 Dedução do Método . . . . . . . . . . . . . . . . . . . . . 19

3.4.3 Método de Pontos Interiores Primal-Dual . . . . . . . . . . . . . . 21

3.4.4 Cálculo Alternativo do Valor do Gap . . . . . . . . . . . . . . . . . 22

3.5 Método de Pontos Interiores Primal-Dual Preditor-Corretor com Variáveis

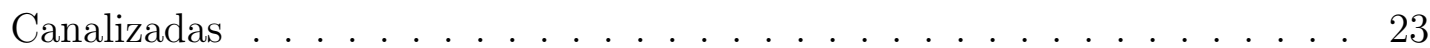

3.5.1 Construção do Método de Pontos Interiores Preditor-Corretor . . . 23

3.5.2 Método de Pontos Interiores Preditor-Corretor . . . . . . . . . . . . 25

3.6 Extensão do Método de Pontos Interiores para o Problema de Programação Quadrática Convexa . . . . . . . . . . . . . . . . 27

3.6 .1 Dedução do Método . . . . . . . . . . . . . . . . . . 27

3.6.2 Critério de Convergência e Valor do Gap . . . . . . . . . . . . . . . 29 
4 Aplicação de Métodos de Pontos Interiores no Problema de Fluxo de Potência 30

4.1 Métodos de Pontos Interiores Aplicados ao Fluxo de Potência Ótimo . . 30

4.1.1 Método de Pontos Interiores Primal-Dual _ . . . . . . . . . . . 30

4.1.2 Método de Pontos Interiores Preditor-Corretor . . . . . . . . . . . . 37

4.1 .3 Utilização de uma Árvore Geradora . . . . . . . . . . . . . . . . . . 41

4.1.4 Formação da Matriz de Reatância . . . . . . . . . . . . . . . . . . 41

4.1.5 Inicialização, Parâmetros e Critério de Convergência . . . . . . . . . . 42

4.2 Métodos de Pontos Interiores Aplicados ao Fluxo de Potência Ótimo Usando o Princípio do Mínimo Esforço . . . . . . . . . . . . . . . . 43

4.2.1 Método de Pontos Interiores Primal-Dual . . . . . . . . . . . . . . . 43

4.2.2 Desenvolvimento Alternativo do Método . . . . . . . . . . . . . . . 48

4.2.3 Método de Pontos Interiores Preditor-Corretor . . . . . . . . . . . . 50

4.2.4 Inicialização, Parâmetros e Critério de Convergência . . . . . . . . . 53

5 Resultados Numéricos $\quad 54$

5.1 Sistemas Testes . . . . . . . . . . . . . . . . 54

5.2 Resultados para o Sistema IEEE30 . . . . . . . . . . . . . . . 55

5.3 Resultados para o Sistema IEEE118 . . . . . . . . . . . . . . . . . 59

5.4 Resultados para os Demais Sistemas . . . . . . . . . . . . . . . . 62

6 Conclusões e Perspectivas Futuras $\quad 65$ 


\section{Lista de Figuras}

2.1 Representação da rede elétrica do Exemplo $2.1 \ldots \ldots$. . . . . . . . . . 5

2.2 Representação em grafo do Exemplo $2.1 \ldots \ldots$. . . . . . . . . . . . 6

2.3 Representação da primeira lei de Kirchhoff na rede do Exemplo 2.1 . . . . 7

2.4 Representação da rede elétrica do Exemplo $2.2 \ldots \ldots$. . . . . . . . . . . 9

5.1 Mapa físico associado ao sistema teste IEEE30 . . . . . . . . . . . . 55

5.2 Mapa físico associado ao sistema teste IEEE118 . . . . . . . . . . . 56

5.3 Sistema IEEE30 . . . . . . . . . . . . . . . . . . . . . . . . . . . 58

5.4 Despacho de potência ativa para ponderações distintas para MFR . . . . . 60

5.5 Despacho de potência ativa para ponderações distintas para PME . . . . . 60 


\section{Lista de Tabelas}

5.1 IEEE30-Carga das barras em MW. . . . . . . . . . . . . . 57

5.2 Valores do desempenho do método para MFR. . . . . . . . . . . . . . 57

5.3 Valores do desempenho do método para PME. . . . . . . . . . . . . . 58

5.4 Valores de geração de potência ativa, em MW, para MFR. . . . . . . . . . 59

5.5 Valores de geração de potência ativa, em MW, para PME. . . . . . . . . . 59

5.6 Resultados obtidos para o sistema IEEE118. . . . . . . . . . . . . . . . . 61

5.7 Valores do desempenho dos métodos para IEEE30. . . . . . . . . . . . 62

5.8 Valores do desempenho dos métodos para IEEE118. . . . . . . . . . . . 63

5.9 Valores do desempenho dos métodos para SSC810 . . . . . . . . . . . 63

5.10 Valores do desempenho dos métodos para SSC1654. . . . . . . . . . . . 64

5.11 Valores do desempenho dos métodos para SSC1732. . . . . . . . . . . 64

5.12 Valores do desempenho dos métodos para BRASIL. . . . . . . . . . . . 64 


\section{Capítulo 1}

\section{Introdução}

No ano de 1984, Karmarkar [9] propôs seu método de pontos interiores, fazendo com que este ano fosse considerado um marco na história da programação linear. Mesmo Karmarkar não tendo apresentado resultados computacionais que comprovassem a eficiência de seu método, outros trabalhos logo demonstraram a eficiência de suas idéias. Em particular, os bons resultados obtidos em [1] deram um impulso ao desenvolvimento desta classe de métodos.

O interesse no desenvolvimento destes métodos se deve ao fato de que, geralmente, problemas reais envolvem a tomada de decisão, visando a minimização de recursos ou a maximização dos lucros.

Muitos destes problemas podem ser modelados usando programação linear. O principal obstáculo fica por conta da dimensão que estes problemas podem assumir. Tais problemas são, muitas vezes, de grande porte, exigindo o uso e desenvolvimento de técnicas especiais para sua resolução. Daí a utilização dos métodos de pontos interiores, uma vez que, desde o seu surgimento, eles têm se mostrado eficientes para a resolução de problemas de grande porte $[1,11,12,17]$.

O objetivo deste trabalho consiste em comparar duas abordagens para a modelagem do problema de fluxo de potência ótimo utilizando os métodos de pontos interiores. Este desenvolvimento procurou explorar as características particulares do problema e também as particularidades da estrutura matricial de modo a obter implementações mais 
eficientes.

O problema de fluxo de potência linearizado DC é representado por um modelo de fluxos em rede com restrições adicionais [2], e aplicamos o princípio do mínimo esforço a esta formulação. Desenvolvemos o método de pontos interiores para este modelo, exploramos a estrutura matricial e comparamos com a implementação desenvolvida em $[20]$.

O problema de fluxo de potência ótimo DC é de grande importância na área de sistemas de potência, servindo como base para diversas outras aplicações. O surgimento dos métodos de pontos interiores trouxe à tona uma nova linha de pesquisa nesta área. Estes métodos são reconhecidos por sua robustez, principalmente devido ao tratamento eficiente de desigualdades [23].

Inicialmente, no Capítulo 2, vamos descrever o problema de fluxo de potência ótimo DC na formulação de fluxos em redes e aplicaremos nele o princípio do mínimo esforço a fim de obter a formulação principal de nosso estudo.

No Capítulo 3, apresentamos os métodos de pontos interiores e construimos um método, de propósito geral, para variáveis canalizadas.

Já no Capítulo 4, desenvolveremos métodos de pontos interiores para os problemas de fluxo de potência ótimo linearizado DC e de fluxo de potência ótimo usando o princípio do mínimo esforço.

O Capítulo 5 apresenta os resultados numéricos da implementação dos métodos desenvolvidos no Capítulo 4 para o problema de fluxo de potência ótimo utilizando os modelos de fluxos em rede e o princípio do mínimo esforço.

Finalmente, o Capítulo 6 apresenta as conclusões do nosso trabalho e as perspectivas futuras. 


\section{Capítulo 2}

\section{O Problema de Fluxo de Potência Ótimo DC}

Neste capítulo, vamos apresentar o modelo matemático a ser utilizado pelo fluxo de potência ótimo DC. O modelo a ser considerado leva em conta a alocação de potência ativa com restrições de geração e transmissão, sendo representado por um modelo de fluxos em redes com restrições adicionais. Depois, vamos introduzir o princípio do mínimo esforço e aplicá-lo ao modelo do fluxo de potência ótimo DC, encontrando, assim, o modelo do nosso problema de estudo. Mas, antes de mostrarmos os modelos matemáticos, vamos fazer uma pequena introdução aos sistemas de potência.

\subsection{Sistemas de Potência}

Sabemos que a energia elétrica tem um importante papel na nossa sociedade, desde a sua utilização para fins domésticos até na indústria, não é possível conceber a falta deste importante insumo em qualquer atividade. Daí a relevância dos estudos relacionados à melhoria de geração e transmissão dessa energia.

Vamos, agora, fornecer um panorama geral sobre o setor energético brasileiro.

Até o ano de 1970, a energia final consumida de origem elétrica no Brasil tinha uma participação no consumo final menor que 20\%. Após o primeiro choque do petróleo, o percentual de consumo final de energia de origem elétrica chegou em $22 \%$ em 
1975, atingindo, em 1999, um percentual de 40\%. Vale lembrar que as hidrelétricas são responsáveis, atualmente, por cerca de $95 \%$ da energia elétrica gerada [16].

Este quadro se deve aos investimentos feitos em hidrelétricas de grande porte. Se, por um lado, estes projetos necessitam de investimentos de grande vulto, por outro, o custo de geração resultante tem sido compensador em relação às outras alternativas.

O sistema brasileiro de geração de energia elétrica conta com características que o fazem único no mundo:

1. Predominância hidrelétrica.

2. Grandes extensões geográficas e grandes distâncias entre as fontes geradoras e os principais centros consumidores.

3. Vários potenciais de aproveitamento no mesmo rio.

4. Diversidade de regimes hidrológicos e pluviométricos.

5. Grau de interligação elétrica entre os sistemas (região sul/sudeste/centro-oeste) relativamente alto em comparação com outros países.

6. Grande potencial hidrelétrico inexplorado.

Com estas características, podemos perceber a importância do planejamento integrado da expansão e utilização do sistema de geração e transmissão.

\subsection{Modelagem Matemática do Problema de Fluxo de Potência Ótimo}

Nesta seção, determinaremos o modelo matemático a ser utilizado para o fluxo de potência ótimo. O modelo a ser considerado leva em conta a alocação de potência ativa com restrições de geração e transmissão. Utilizaremos, neste trabalho, um modelo de fluxo de potência linearizado DC, isto é, fluxo de carga com corrente contínua, com limites no fluxo de carga ativo. 
Para construirmos o modelo, consideramos os elementos existentes no sistema, que são, basicamente, os geradores, as cargas e as linhas de transmissão que permitem o transporte de energia.

Visando a melhor compreensão de cada etapa do processo de construção, utilizamos um pequeno exemplo de uma rede elétrica.

Exemplo 2.1 Considere uma rede elétrica com duas barras de geração que podem representar usinas hidrelétricas ou termoelétricas. Temos, também seis barras de carga que podem representar o consumo das cidades ou de grandes indústrias. Uma possível representação deste problema é dada na Figura 2.1.

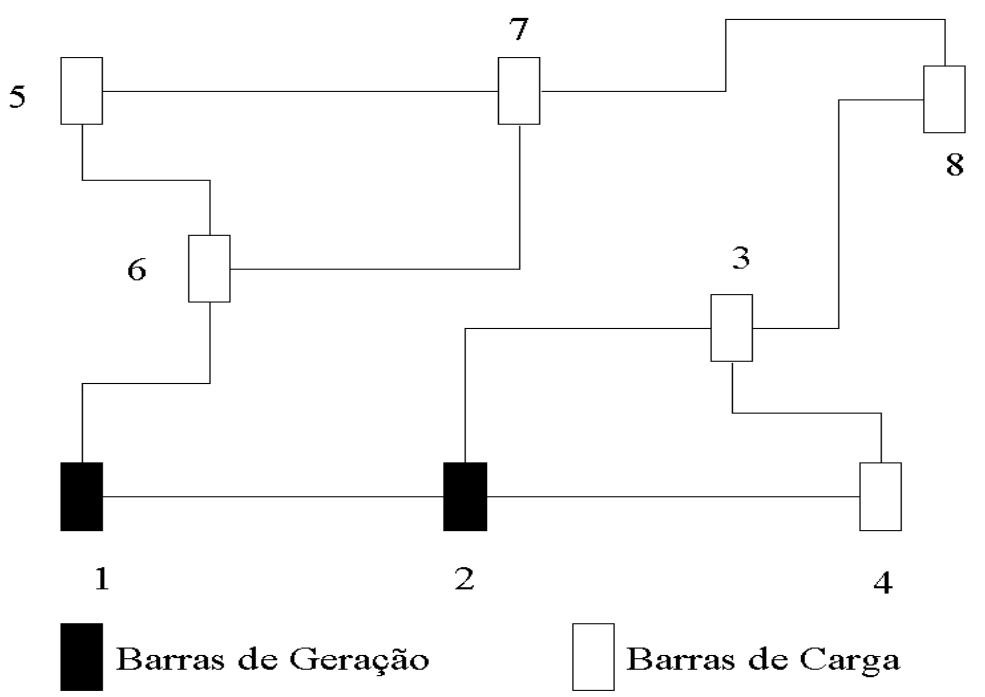

Figura 2.1: Representação da rede elétrica do Exemplo 2.1

Neste problema, a representação por grafos é intuitiva. Assim, a Figura 2.1 pode ser representada pelo grafo dado na Figura 2.2.

Portanto, na representação em grafos, temos que:

- Nós: representam as barras de geração ou as barras de carga.

- Arcos: representam as linhas de transmissão.

Vamos considerar também as dimensões do problema, que são: 


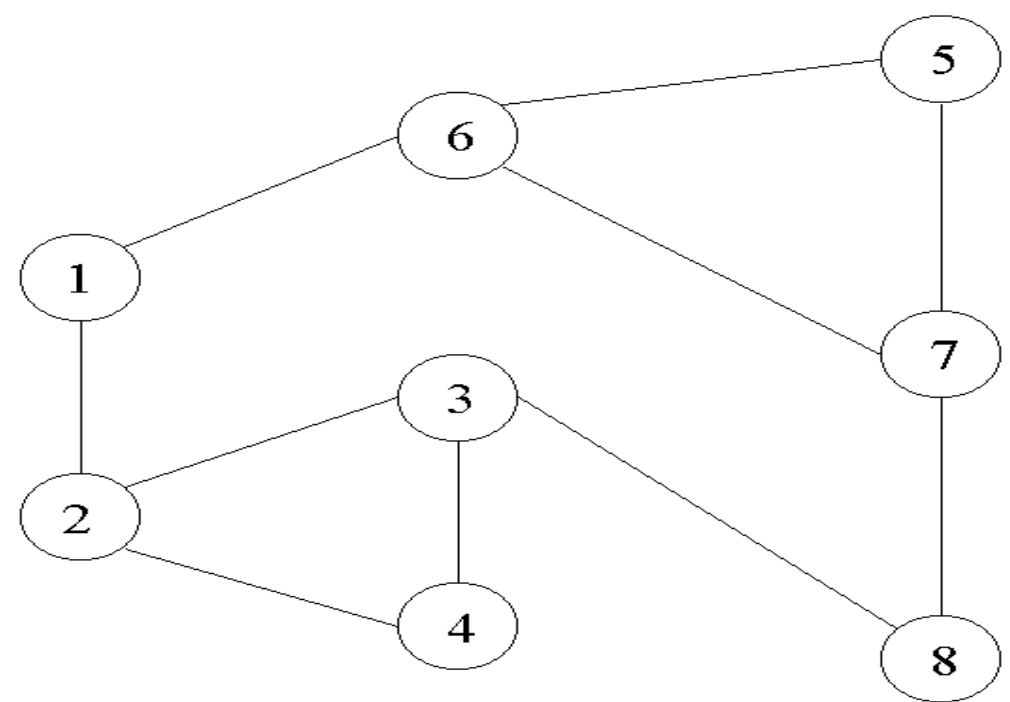

Figura 2.2: Representação em grafo do Exemplo 2.1

- $m$ : número de nós do grafo;

- $n$ : número de arcos do grafo;

- $c$ : número de ciclos canônicos do grafo.

Utilizando o grafo da Figura 2.2 e a segunda lei de Kirchhoff, temos a equação $(2.1)$.

$$
A f=p-l,
$$

onde: $A$ representa a matriz de incidência nó-arco de dimensão $m \times n, f$ representa o valor do fluxo de potência em cada linha de transmissão de dimensão $n \times 1, p$ representa a geração de potência em cada barra de geração de dimensão $m \times 1$ e $l$ representa a demanda por potência em cada barra de carga de dimensão $m \times 1$.

A equação (2.1) expressa o balanço de potência nos nós da rede elétrica, ou seja, a injeção de potência líquida em cada nó deve ser igual ao fluxo líquido que escoa pelos arcos que se conectam a ele.

Além do atendimento da demanda de potência, devemos levar em consideração que a soma das tensões em cada um dos percursos fechados existentes na rede elétrica 
deve ser igual a zero, ou seja, a primeira lei de Kirchhoff, conforme a equação (2.2).

$$
T f=0,
$$

onde: $T$ representa a matriz de reatâncias de dimensão $c x n$.

Esta situação pode ser melhor visualizada na Figura 2.3.

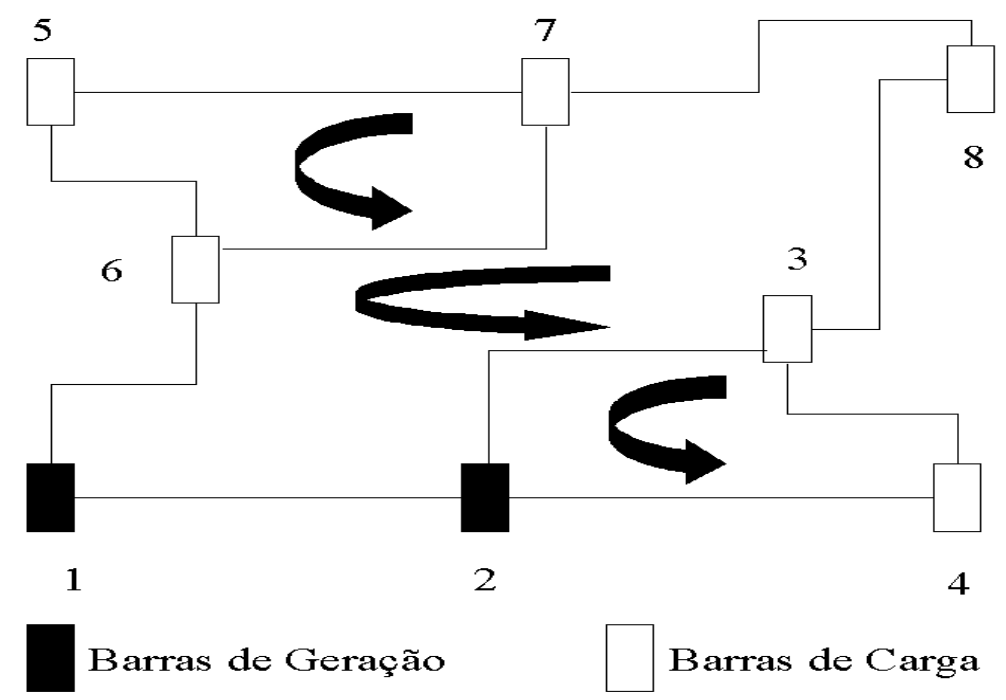

Figura 2.3: Representação da primeira lei de Kirchhoff na rede do Exemplo 2.1

Além disso, devemos considerar os limites de geração e transmissão de energia elétrica que são representados pelas equações (2.3) e (2.4), respectivamente.

$$
\begin{gathered}
p^{\text {min }} \leq p \leq p^{\max } \\
f^{\text {min }} \leq f \leq f^{\text {max }}
\end{gathered}
$$

onde: $p^{\text {min }}$ e $p^{\max }$ representam os valores mínimo e máximo de $p$, respectivamente, e $f^{\text {min }}$ e $f^{\max }$ representam os valores mínimo e máximo de $f$, respectivamente.

Por último, utilizamos uma função objetivo que forneça um critério para a determinação dos valores ótimos dos fluxos de potência $f$ e dos valores de geração $p$ expressos na equação (2.5).

$$
\min \quad \alpha f^{t} R f+\beta\left(p^{t} H p+c^{t} p\right)
$$

onde: $f^{t} R f$ representa a parcela referente ao valor econômico das perdas na transmissão, $R$ representa a matriz diagonal associada às resistências das linhas de transmissão de 
dimensão $n \times n,\left(p^{t} H p+c^{t} p\right)$ representa a parcela referente ao custo de geração, no caso de termoelétricas ou perdas de geração, no caso de hidrelétricas [22], $H$ representa a matriz diagonal dos coeficientes do termo quadrático de geração de dimensão $m \times m, c$ representa o vetor dos coeficientes lineares de dimensão $m \times 1$, e $\alpha$ e $\beta$ são pesos atribuídos à geração e à transmissão.

Portanto, o modelo matemático do problema de fluxo de potência ótimo DC a ser considerado é dado por (2.6):

$$
\begin{aligned}
& \min \quad \alpha f^{t} R f+\beta\left(p^{t} H p+c^{t} p\right) \\
& \text { sa } \quad A f=p-l, T f=0 \\
& f^{\min } \leq f \leq f^{\max }, p^{\min } \leq p \leq p^{\max }
\end{aligned}
$$

Este é um problema quadrático com um conjunto de restrições lineares, onde as equações em (2) representam a rede de geração - transmissão e as equações (3) representam as capacidades de geração e transmissão do sistema.

\subsection{O Princípio do Mínimo Esforço}

O princípio do mínimo esforço consiste na simplificação do modelo de fluxo de potência ativa linearizado(2.6), correspondente a um fluxo em redes, antes da aplicação de um método de otimização.

Temos a relação $f=X^{-1} A^{t} \theta$, onde:

$X$ representa a matriz diagonal das reatâncias das linhas de dimensão $n \times n$;

$\theta$ representa o vetor dos ângulos das tensões de dimensão $m \times 1$.

Esta relação assegura a primeira lei de Kirchhoff, pois se tivéssemos na função objetivo $f^{t} X f$, já estaría garantido que $T f=0$, desde que nenhuma variável de fluxo esteja ativa. Substituindo $f$ na segunda lei de Kirchhoff (primeira equação de (2)), temos:

$$
\begin{aligned}
A f & =p-l \Longrightarrow A X^{-1} A^{t} \theta=p-l \\
& \Longrightarrow A X^{-1} A^{t} \theta-p=-l .
\end{aligned}
$$

Fazendo $B=A X^{-1} A^{t}$, obtemos:

$$
B \theta-p=-l
$$


Note que $B$ é uma matriz quadrada $(m \mathrm{x} m)$, que só depende dos dados de rede e pode ser expressa como

$$
b_{i j}=\left\{\begin{aligned}
\sum_{(i, j) \in \Omega} \frac{1}{x_{i j}}, & \text { para } i=j ; \\
-\frac{1}{x_{i j}}, & \text { se } i \neq j \text { e }(i, j) \in \Omega ; \\
0, & \text { caso contrário, }
\end{aligned}\right.
$$

$B$ é chamada matriz de susceptância da rede e $\Omega$ é o conjunto de linhas da rede, ou seja, dos arcos do grafo associado à rede com orientação definida.

Para uma melhor visualização das matrizes envolvidas meste estudo, vejamos um exemplo.

Exemplo 2.2 Considere uma rede elétrica com três barras e três linhas de transmissão, representada pela Figura 2.4 .

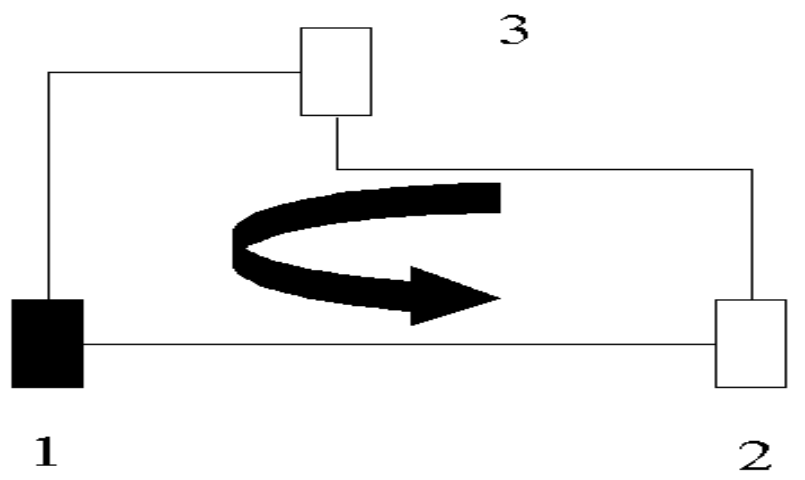

Figura 2.4: Representação da rede elétrica do Exemplo 2.2

O conjunto $\Omega$, para este exemplo é dado por:

$$
\Omega=\{(1,2),(2,3),(3,1)\}
$$

Temos que a matriz de reatâncias é:

$$
T=\left[\begin{array}{lll}
t_{12} & t_{23} & t_{31}
\end{array}\right] .
$$

A matriz incidência nó-arco é dada por:

$$
A=\left[\begin{array}{ccc}
1 & 0 & -1 \\
-1 & 1 & 0 \\
0 & -1 & 1
\end{array}\right]
$$


Considere a matriz $X$ das reatâncias das linhas dada abaixo:

$$
X=\left[\begin{array}{ccc}
x_{12} & 0 & 0 \\
0 & x_{23} & 0 \\
0 & 0 & x_{31}
\end{array}\right]
$$

Então, como $B=A X^{-1} A^{t}$, temos:

$$
\begin{gathered}
B=\left[\begin{array}{ccc}
1 & 0 & -1 \\
-1 & 1 & 0 \\
0 & -1 & 1
\end{array}\right]\left[\begin{array}{ccc}
\frac{1}{x_{12}} & 0 & 0 \\
0 & \frac{1}{x_{23}} & 0 \\
0 & 0 & \frac{1}{x_{31}}
\end{array}\right]\left[\begin{array}{ccc}
1 & -1 & 0 \\
0 & 1 & -1 \\
-1 & 0 & 1
\end{array}\right] \Longrightarrow \\
B=\left[\begin{array}{ccc}
\frac{1}{x_{12}}+\frac{1}{x_{31}} & \frac{-1}{x_{12}} & \frac{-1}{x_{31}} \\
\frac{-1}{x_{12}} & \frac{1}{x_{12}}+\frac{1}{x_{23}} & \frac{-1}{x_{23}} \\
\frac{-1}{x_{31}} & \frac{-1}{x_{23}} & \frac{1}{x_{23}}+\frac{1}{x_{31}}
\end{array}\right],
\end{gathered}
$$

o que condiz com a definição dada pela equação (2.7).

A matriz $B$ é singular, pois a soma das componentes $b_{i j}, i \neq j$, de uma linha $i$ em $B$ é igual ao elemento $b_{i i}$ da diagonal principal. Para resolver este problema, elimina-se uma das linhas $(i)$ de $B$ e adota-se a barra correspondente como referência angular $\left(\theta_{i}=0\right)$, ou seja, eliminamos a linha e a coluna correspondente a $\theta_{i}$ de B.

Consideremos, a partir de agora, $B$ como sendo uma matriz não-singular.

É necessário considerar também os limites de transmissão que com a substituição se transformam em $f_{i j}^{\min } x_{i j} \leq \theta_{i}-\theta_{j} \leq f_{i j}^{\max } x_{i j}$. De fato, sabemos que $A^{t} \theta$ nada mais é que $\theta_{i}-\theta_{j}$ e que a inversa da matriz $X$ é a matriz cujos elementos da diagonal são o inverso dos elementos da diagonal de $X$. Essa substituição transforma variáveis canalizadas em restrições canalizadas. Esta pode ser uma desvantagem considerável dependendo do método de otimização utilizado, sendo estas restrições usualmente desconsideradas numa primeira resolução do problema, e apenas aquelas violadas são acrescidas ao modelo para a próxima otimização. Este processo é repetido até não existir restrições violadas. Como os métodos de pontos interiores são considerados robustos no tratamento de desigualdades, espera-se que esta desvantagem não seja tão acentuada nesta abordagem. 
Já o termo referente ao fluxo de potência ativa da função objetivo se reduz a uma função do ângulo das tensões, ou seja:

$$
\begin{aligned}
f^{t} R f & =\left(X^{-1} A^{t} \theta\right)^{t} R\left(X^{-1} A^{t} \theta\right) \\
& =\theta^{t} A X^{-t} R X^{-1} A^{t} \theta .
\end{aligned}
$$

Fazendo $\tilde{B}=A X^{-t} R X^{-1} A^{t}$, obtemos:

$$
f^{t} R f=\theta^{t} \tilde{B} \theta
$$

A matriz $\tilde{B}$ é uma matriz quadrada $(m \mathrm{x} m)$, não singular e, assim como $B$, só depende dos dados da rede.

Portanto, podemos escrever o modelo matemático para o problema de fluxo de potência ótimo baseado no princípio do mínimo esforço como segue:

$$
\begin{array}{cc}
\min & \theta^{t} \tilde{B} \theta+p^{t} H p+c^{t} p \\
\text { sa } & B \theta-p=-l \\
& p^{\text {min }} \leq p \leq p^{\text {max }} .
\end{array}
$$




\section{Capítulo 3}

\section{Métodos de Pontos Interiores}

Neste Capítulo, apesentaremos os métodos de pontos interiores e construiremos um método, de propósito geral, para variáveis canalizadas. Depois, introduziremos o método preditorcorretor, e o mesmo será desenvolvido para um problema de programação linear.

\subsection{Conceitos Iniciais sobre Pontos Interiores}

Antes de descrevermos os métodos de pontos interiores, devemos caracterizar o que é um problema de programação linear e o que é um ponto interior em um problema desse tipo, respectivamente. Os problemas de programação linear derivam da construção de uma representação matemática para um problema real em que se quer minimizar ou maximizar uma função objetivo linear, ao mesmo tempo em que as variáveis estão sujeitas a determinadas restrições também lineares. Um ponto interior é aquele em que todas as variáveis se encontram estritamente dentro de seus limites.

Vamos considerar o problema de programação linear, na forma padrão, dado por:

$$
\begin{array}{cc}
\text { min } & c^{t} x \\
\text { sa } & A x=b \\
& x \geq 0 .
\end{array}
$$

Esta formulação é denominada problema primal e está associada ao problema dual 


$$
\begin{array}{cc}
\max & b^{t} y \\
\text { sa } & A^{t} y+z=c \\
& y \text { livre, } z \geq 0 .
\end{array}
$$

Pela definição de ponto interior, então, $\left\{x^{0} \mid x^{0}>0\right\}$ é um ponto interior para o problema primal, assim como $\left\{y^{0}, z^{0} \mid z^{0}>0\right\}$ é um ponto interior para o problema dual.

Temos também as condições de otimalidade. Para uma melhor caracterização, levemos em conta as duas formulações descritas em (3.1) e (3.2). Se um dado ponto $(x, y, z)$ é ótimo para os problemas primal e dual, então, as seguintes condições deverão ser satisfeitas [26]:

1. Factibilidade Primal: $b-A x=0, x \geq 0$.

2. Factibilidade Dual: $c-A^{t} y-z=0, z \geq 0$.

3. Complementaridade: $x_{i} z_{i}=0, i=1, \ldots, n$, onde $n$ é a dimensão dos vetores $x$ e $z$.

Um outro conceito, também importante, e que será utilizado para a construção de métodos de pontos interiores é o gap $(\gamma)$. O gap é a diferença entre os valores das funções objetivo do problema primal e o seu dual. No caso das formulações dadas por (3.1) e (3.2), temos: $\gamma=c^{t} x-b^{t} y=x^{t} z$, se o ponto $(x, y, z)$ for primal e dual factíveis.

\subsection{Usando o Método de Newton para Construir Métodos de Pontos Interiores}

A essência de um método de pontos interiores consiste em se aplicar o método de Newton às condições de otimalidade de um problema de programação linear. Para tanto, vamos rever o funcionamento do método de Newton para uma variável e, a seguir, para várias variáveis, de modo a aplicar este último às condições de otimalidade das formulações dadas em (3.1) e (3.2), e obter um método de pontos interiores. 


\section{- Método de Newton para uma variável:}

Seja $x \in R$ tal que $\phi(x)=0$. Para encontrarmos o valor de $x$ utilizamos sucessivas aproximações da função $\phi(x)$ em torno dos pontos $x^{0}, x^{1}, \ldots, x^{k}$, até que o ponto $x^{k}$ seja tal que $\phi\left(x^{k}\right) \approx 0$. A aproximação inicial que utilizamos é dada pela fórmula de Taylor em torno de $x^{0}$ :

$$
\phi(x)=\phi\left(x^{0}\right)+\phi^{\prime}\left(x^{0}\right)\left(x-x^{0}\right)+\phi^{\prime \prime}\left(x^{0}\right)\left(x-x^{0}\right)^{2} / 2 !+\ldots
$$

Fazendo a aproximação linear:

$$
\phi(x) \approx \phi\left(x^{0}\right)+\phi^{\prime}\left(x^{0}\right)\left(x^{1}-x^{0}\right)=0 \Longrightarrow x^{1}=x^{0}-\phi\left(x^{0}\right) / \phi^{\prime}\left(x^{0}\right) .
$$

Podemos aplicar esta fórmula para obter $x^{1}$ e depois aplicar o valor de $x^{k}$ sucessivamente de modo a obter novos valores, até que $\phi\left(x^{k}\right) \simeq 0$. Construímos, assim, o método de Newton para uma variável.

\section{Método de Newton para uma variável}

Dado $x^{0}$.

Para $k=0,1, \ldots$, faça

$d^{k}=-\phi\left(x^{k}\right) / \phi^{\prime}\left(x^{k}\right)$

$x^{k+1}=x^{k}+d^{k}$

Até convergir.

\section{- Método de Newton para várias variáveis:}

Seja $x \in \Re^{n}$ tal que $\phi_{i}(x)=0$, para $i=1,2, \ldots, n$. Novamente, para encontrarmos o valor de $x$ adequado utilizamos sucessivas aproximações das funções $\phi(x)$ em torno dos pontos $x^{0}, x^{1}, \ldots, x^{k}$, até que o ponto $x^{k}$ seja tal que $\phi_{i}\left(x^{k}\right) \simeq 0$. A diferença em relação ao método de Newton para uma variável se dá na aproximação que é fornecida pela fórmula de Taylor para várias variáveis:

$$
\phi_{i}(x)=\phi_{i}\left(x^{0}\right)+\left(\nabla \phi_{i}\left(x^{0}\right)\right)^{t}\left(x-x^{0}\right)+\ldots,
$$

onde $\left(\nabla \phi_{i}\left(x^{0}\right)\right)^{t}=\left[\delta \phi_{i}\left(x^{0}\right) / \delta x_{1}, \ldots, \delta \phi_{i}\left(x^{0}\right) / \delta x_{n}\right]$. 
Fazendo a aproximação linear:

$0=\phi_{i}\left(x^{0}\right)+\left(\nabla \phi_{i}\left(x^{0}\right)\right)^{t}\left(x^{1}-x^{0}\right), i=1, \ldots, n$

$\left(\nabla \phi_{i}\left(x^{0}\right)\right)^{t}\left(x^{1}-x^{0}\right)=-\phi_{i}\left(x^{0}\right), i=1, \ldots, n$.

Seja a matriz Jacobiana: $J\left(x^{0}\right)=\left(\begin{array}{c}\left(\nabla \phi_{1}\left(x^{0}\right)\right)^{t} \\ \ldots \\ \left(\nabla \phi_{n}\left(x^{0}\right)\right)^{t}\end{array}\right)$ e $f\left(x^{0}\right)=\left(\begin{array}{c}\phi_{1}\left(x^{0}\right) \\ \ldots \\ \phi_{n}\left(x^{0}\right)\end{array}\right)$, então:

$J\left(x^{0}\right)\left(x^{1}-x^{o}\right)=-f\left(x^{0}\right)=-\left(\begin{array}{c}\phi_{1}\left(x^{0}\right) \\ \cdots \\ \phi_{n}\left(x^{0}\right)\end{array}\right)$.

$x^{1}=x^{0}-\left(J\left(x^{0}\right)\right)^{-1} f\left(x^{0}\right)$.

\section{Método de Newton para várias variáveis}

Dado $x^{0} \in \Re$.

Para $k=0,1, \ldots$, faça

$d^{k}=-\left(J\left(x^{k}\right)\right)^{-1} f\left(x^{k}\right)$

$x^{k+1}=x^{k}+d^{k}$

Até convergir.

Tendo em vista as condições de otimalidade na Seção 3.1 e o Método de Newton para múltiplas variáveis, o próximo passo consiste em obter um método de pontos interiores.

Consideremos o problema com formulação primal e dual fornecida por (3.1) e (3.2), respectivamente. A idéia para se construir um método de pontos interiores consiste em se aplicar o método de Newton ao sistema $f(x, y, z)=0$ formado pelas condições de otimalidade, resolvendo os problemas primal e dual simultaneamente. Temos que $f(x, y, z)$ é dada por:

$$
f\left(x^{0}, y^{0}, z^{0}\right)=\left(\begin{array}{c}
A x^{0}-b \\
A^{t} y^{0}+z^{0}-c \\
X^{0} Z^{0} e
\end{array}\right)=-\left(\begin{array}{c}
r_{p}{ }^{0} \\
r_{d}{ }^{0} \\
r_{a}{ }^{0}
\end{array}\right)=\left(\begin{array}{c}
f_{p} \\
f_{d} \\
f_{a}
\end{array}\right)
$$

onde $X^{0}=\operatorname{diag}\left(x^{0}\right), Z^{0}=\operatorname{diag}\left(z^{0}\right)$, e representa o vetor unitário e $X^{0} Z^{0} e=0$ é equivalente a $x_{i}{ }^{0} z_{i}{ }^{0}=0, i=1, \ldots, n$. 
Dado um ponto $\left(x^{0}, y^{0}, z^{0}\right)$ e desconsiderando as restrições $x \geq 0$ e $z \geq 0$, calculamos o ponto $(x, y, z)$ utilizando o método de Newton para várias variáveis:

$$
\left(x^{1}, y^{1}, z^{1}\right)=\left(x^{0}, y^{0}, z^{0}\right)-\left(J\left(x^{0}, y^{0}, z^{0}\right)\right)^{-1} f\left(x^{0}, y^{0}, z^{0}\right)
$$

onde $J\left(x^{0}, y^{0}, z^{0}\right)=\left(\begin{array}{c}\nabla f_{p} \\ \nabla f_{d} \\ \nabla f_{a}\end{array}\right)=\left(\begin{array}{ccc}A & 0 & 0 \\ 0 & A^{t} & I \\ Z & 0 & X\end{array}\right)$.

Assim, $d$ será dado por:

$$
d^{0}=\left(\begin{array}{ccc}
A & 0 & 0 \\
0 & A^{t} & I \\
Z^{0} & 0 & X^{0}
\end{array}\right)^{-1}\left(\begin{array}{c}
r_{p}^{0} \\
r_{d}^{0} \\
r_{a}^{0}
\end{array}\right)=\left(\begin{array}{c}
d x \\
d y \\
d z
\end{array}\right) .
$$

Podemos resumir estes cálculos em um algoritmo como se segue:

\section{Método primal-dual afim-escala [14]}

Dado um ponto $\left(x^{0}, y^{0}, z^{0}\right)$ interior, ou seja, $\left(x^{0}, z^{0}\right)>0$.

Para $k=0,1, \ldots$, faça

$r_{p}^{k}=b-A x^{k}$

$r_{d}^{k}=c-A^{t} y^{k}-z^{k}$

$r_{a}^{k}=-X^{k} Z^{k} e$

$d^{k}=\left(\begin{array}{c}d x^{k} \\ d y^{k} \\ d z^{k}\end{array}\right)=\left(\begin{array}{ccc}A & 0 & 0 \\ 0 & A^{t} & I \\ Z^{0} & 0 & X^{0}\end{array}\right)^{-1}\left(\begin{array}{c}r_{p}{ }^{k} \\ r_{d}{ }^{k} \\ r_{a}{ }^{k}\end{array}\right)$

Calculamos o tamanho do passo $\alpha_{p}{ }^{k}$ e $\alpha_{d}^{k}$, tal que $x^{k+1}>0$ e $z^{k+1}>0$.

$x^{k+1}=x^{k}+\alpha_{p}^{k} d x^{k}$

$y^{k+1}=y^{k}+\alpha_{d}^{k} d y^{k}$

$z^{k+1}=z^{k}+\alpha_{d}^{k} d z^{k}$

Até convergir.

Ou seja, aplica-se o método de Newton às condições de otimalidade e caminha-se na direção encontrada, mantendo as variáveis não-negativas interiores.

Uma observação importante é que dados $x^{0}$ e $z^{0}$ interiores, o tamanho do passo $\alpha$ é calculado de forma que $x^{k+1}$ e $z^{k+1}$ permaneçam interiores. Com $y^{k+1}$ não nos pre- 
ocupamos, pois y é uma variável livre. Levando em conta essa afirmação, temos que os tamanhos dos passos primal e dual são:

$$
\begin{aligned}
\alpha_{p}^{k} & =\min \left\{1, \tau \rho_{p}\right\} \\
\alpha_{d}^{k} & =\min \left\{1, \tau \rho_{d}\right\},
\end{aligned}
$$

onde:

$$
\begin{aligned}
& \rho_{p}=\min _{d x_{i}<0}\left\{-\frac{x_{i}}{d x_{i}}\right\} \\
& \rho_{d}=\min _{d z_{i}<0}\left\{-\frac{z_{i}}{d z_{i}}\right\} .
\end{aligned}
$$

Adota-se o passo máximo $\alpha=1$ porque este é o tamanho de passo natural no Método de Newton e $\alpha=1$ pode levar a um ponto factível em uma iteração.

\subsection{Método de Pontos Interiores Primal-Dual}

Vimos na seção 3.2 como obter um método de pontos interiores primal-dual afim-escala [14]. Este método, porém, tem uma grande desvantagem, pois permite que os pontos calculados $(x, z)$ se aproximem de seus limites muito rapidamente.

Consequentemente, as direções calculadas perto destes limites são muito distorcidas, pois o valor de $x_{i} z_{i}$ se torna próximo de zero rapidamente e o método progride lentamente, podendo inclusive não convergir.

Para evitar que isto aconteça, acrescentamos a cada iteração uma perturbação $\mu^{k}$ na condição de complementaridade. No caso do problema estudado, teríamos $x_{i} z_{i}=0$, tal que o método primal-dual deverá encontrar a solução do seguinte sistema:

$$
\left\{\begin{array}{c}
b-A x=0 \\
c-A^{t} y-z=0 \\
\mu e-X Z e=0
\end{array}\right.
$$

Assim, o desenvolvimento dos métodos a seguir tem o propósito de não somente construir o método de pontos interiores para um problema de programação linear que tenha restrições de igualdade e desigualdade, bem como variáveis canalizadas, mas, também, 
utilizar uma perturbação na condição de complementaridade de modo a tornar o método mais eficiente.

Para isto, vamos descrever os problemas primal e dual de modo que com estas informações consigamos escrever as condições de otimalidade e, a partir delas, desenvolver o método de pontos interiores primal-dual.

\subsection{Método de Pontos Interiores Primal-Dual com Variáveis Canalizadas}

Nesta seção, veremos como construir um método de ponto interior de propósito geral que leve em consideração um problema de programação linear com restrições de igualdade e variáveis canalizadas.

\subsubsection{Formulações Primal e Dual}

Consideremos o problema de minimização dado por:

$$
\begin{array}{ccrl}
\text { min } & f(x) & =c^{t} x \\
\text { sa } & A x & =b \\
& x^{\text {min }} \leq x \leq x^{\text {max }} .
\end{array}
$$

Colocaremos o problema num formato em que o limite inferior seja nulo, fazendo a seguinte mudança de variáveis:

$$
\tilde{x}=x-x^{\min } \Rightarrow x=\tilde{x}+x^{\min } .
$$

Utilizando (3.4) na formulação proposta em (3.3), temos:

$$
\begin{array}{cc}
\min & f(x)=c^{t} \tilde{x}+c^{t} x^{\min } \\
s a & A \tilde{x}=\tilde{b} \\
& 0 \leq \tilde{x} \leq \tilde{u},
\end{array}
$$

onde $\tilde{b}=b-A x^{\min }$ e $\tilde{u}=x^{\max }-x^{\min }$. 
Adicionando as variáveis de folga ao problema, simplificando a notação eliminando os "tils", temos:

$$
\begin{array}{cc}
\text { min } & f(x)=c^{t} x \\
\text { sa } & A x=b \\
& x+t=u \\
x, t \geq 0 .
\end{array}
$$

A partir da formulação primal dada em (3.6), podemos escrever o problema dual como em (3.7), já com as variáveis de folga introduzidas [26].

$$
\begin{array}{cl}
\max & f(x)=b^{t} y-u^{t} w \\
\text { sa } & A^{t} y-w+z=c \\
& w, z \geq 0, y \text { livre. }
\end{array}
$$

\subsubsection{Dedução do Método}

De posse das formulações primal e dual do problema iremos obter as condições de otimalidade que são dadas por:

factibilidade primal: $\left\{\begin{array}{c}b-A x=0 \\ u-x-t=0 \\ (x, t) \geq 0,\end{array}\right.$
factibilidade dual: $\left\{\begin{array}{c}c-A^{t} y+w-z=0 \\ (w, z) \geq 0 \\ y \text { livre, }\end{array}\right.$

condições de complementaridade: $\left\{\begin{array}{c}X Z e=0 \\ T W e=0 .\end{array}\right.$

Aplicando o método de Newton às condições de otimalidade, obtemos:

$$
\left[\begin{array}{ccccc}
A & 0 & 0 & 0 & 0 \\
I & 0 & I & 0 & 0 \\
0 & A^{t} & 0 & -I & I \\
Z & 0 & 0 & 0 & X \\
0 & 0 & W & T & 0
\end{array}\right]\left[\begin{array}{c}
d x \\
d y \\
d t \\
d w \\
d z
\end{array}\right]=\left[\begin{array}{c}
r_{p} \\
r_{u} \\
r_{d} \\
r_{c} \\
r_{e}
\end{array}\right]
$$


onde:

$$
\left\{\begin{array}{rlc}
r_{p} & = & b-A x \\
r_{u} & = & u-x-t \\
r_{d} & = & c-A^{t} y+w-z \\
r_{c} & = & \mu e-X Z e \\
r_{e} & = & \mu e-T W e
\end{array}\right.
$$

Reescrevendo o sistema linear, temos:

$$
\left\{\begin{array}{c}
A d x=r_{p} \\
d x+d t=r_{u} \\
A^{t} d y-d w+d z=r_{d} \\
Z d x+X d z=r_{c} \\
T d w+W d t=r_{e} .
\end{array}\right.
$$

Utilizando a segunda equação de (3.8), temos:

$$
d t=r_{u}-d x
$$

Considerando que $Z, X, T$ e $W$ são matrizes diagonais com elementos positivos, e portanto inversíveis, então, com as duas últimas equações de (3.8) chegamos em:

$$
\left\{\begin{array}{c}
d z=X^{-1}\left(r_{c}-Z d x\right) \\
d w=T^{-1}\left(r_{e}-W d t\right) .
\end{array}\right.
$$

Agora, aplicando (3.10) na terceira equação de (3.8):

$$
A^{t} d y+T^{-1} W d t-X^{-1} Z d x=r_{d}+T^{-1} r_{e}-X^{-1} r_{c}=r_{k}
$$

Substituindo (3.9) em (3.11), temos:

$$
A^{t} d y-\left(T^{-1} W+X^{-1} Z\right) d x=r_{k}-T^{-1} W r_{u}=r_{m}
$$

Assim, nosso sistema é simplificado para:

$$
\left\{\begin{array}{c}
A d x=r_{p} \\
A^{t} d y-D d x=r_{m} .
\end{array}\right.
$$


A matriz $D=\left(T^{-1} W+X^{-1} Z\right)$ pode ser facilmente invertida, pois ela é a soma de matrizes diagonais definidas positivas. Então, podemos eliminar $d x$ da segunda equação de (3.13), e substituindo $r_{m}$ e $r_{k}$, temos:

$$
d x=D^{-1}\left(A^{t} d y-r_{d}-T^{-1} r_{e}+X^{-1} r_{c}+T^{-1} W r_{u}\right)
$$

Substituindo $d x$ na primeira equação de (3.13), temos:

$$
\left(A D^{-1} A^{t}\right) d y-r_{p}=A D^{-1}\left(r_{d}+T^{-1} r_{e}-X^{-1} r_{c}-T^{-1} W r_{u}\right)
$$

Cabe destacar que a inversão de $A D^{-1} A^{t}$ envolve a maior parte do esforço computacional dos métodos de pontos interiores. Mas, essa matriz é simétrica e definida positiva e, na prática, utilizamos a decomposição de Cholesky [6] para resolver o sistema linear.

\subsubsection{Método de Pontos Interiores Primal-Dual}

Tendo obtido a equação (3.15), podemos resumir o método de pontos interiores com variáveis canalizadas e restrições de igualdade:

Dados $\left(x^{0}, t^{0}, w^{0}, z^{0}\right) \geq 0, y^{0}$ livre, $\sigma \in(0,1)$ e $\tau \in(0,1)$.

Para $k=0,1, \ldots$, faça:

$\mu^{k}=\sigma \frac{\gamma^{k}}{n_{p}}$, onde: $n_{p}=$ dimensão do vetor $(x, t)$, e $\gamma^{k}$ é o $g a p$.

Calcule os resíduos $r_{p}^{k}, r_{u}^{k}, r_{d}^{k}, r_{c}^{k}$ e $r_{e}^{k}$.

$$
\begin{aligned}
D^{k} & =\left(T^{k}\right)^{-1} W^{k}+\left(X^{k}\right)^{-1} Z^{k} \\
d y^{k} & =\left(A\left(D^{k}\right)^{-1} A^{t}\right)^{-1}\left(r_{p}{ }^{k}+A\left(D^{k}\right)^{-1}\left(r_{d}^{k}+\left(T^{k}\right)^{-1} r_{e}{ }^{k}-\left(X^{k}\right)^{-1} r_{c}{ }^{k}-\left(T^{k}\right)^{-1} W^{k} r_{u}{ }^{k}\right)\right) \\
d x & =\left(D^{k}\right)^{-1}\left(A^{t} d y^{k}-r_{d}^{k}-\left(T^{k}\right)^{-1} r_{e}{ }^{k}+\left(X^{k}\right)^{-1} r_{c}{ }^{k}+\left(T^{k}\right)^{-1} W^{k} r_{u}{ }^{k}\right) \\
d t^{k} & =r_{u}{ }^{k}-d x^{k} \\
d z^{k} & =\left(X^{k}\right)^{-1}\left(r_{c}{ }^{k}-Z^{k} d x^{k}\right) \\
d w^{k} & =\left(T^{k}\right)^{-1}\left(r_{e}^{k}-W^{k} d t^{k}\right) \\
\rho_{p}^{k} & =\min \left\{\frac{-1}{\min _{i}\left(\frac{d x^{k}}{x_{i}^{k}}\right)}, \frac{-1}{\min _{i}\left(\frac{d t^{k}}{t_{i}^{k}}\right)}\right\} \\
\rho_{d}^{k} & =\min \left\{\frac{-1}{\min _{i}\left(\frac{d w^{k}}{w_{i}^{k}}\right)}, \frac{-1}{\min _{i}\left(\frac{d z^{k}}{z_{i}^{k}}\right)}\right\}
\end{aligned}
$$




$$
\begin{aligned}
\alpha_{p}^{k} & =\min \left(1, \tau \rho_{p}^{k}\right) \\
\alpha_{d}^{k} & =\min \left(1, \tau \rho_{d}^{k}\right) \\
x^{k+1} & =x^{k}+\alpha^{p} d x^{k} \\
t^{k+1} & =t^{k}+\alpha^{p} d t^{k} \\
y^{k+1} & =y^{k}+\alpha^{d} d y^{k} \\
z^{k+1} & =z^{k}+\alpha^{d} d z^{k} \\
w^{k+1} & =w^{k}+\alpha^{d} d w^{k}
\end{aligned}
$$

Até convergir.

Na prática, utilizamos $\sigma=\frac{1}{\sqrt{n}}$ e $\tau=0,99995$ [12].

\subsubsection{Cálculo Alternativo do Valor do Gap}

Com o propósito de simplificação dos cálculos a serem efetuados pelo método apresentado na seção 3.4.3, vamos provar a equivalência de uma fórmula mais simples para o valor do gap. Ou seja, vamos mostrar que o gap dado por $\gamma=c^{t} x-b^{t} y+u^{t} w$ equivale a $\gamma=x^{t} z+t^{t} w$, para todo ponto factível.

Seja

(i) $x^{t} z+t^{t} x$;

Temos que $(x, t, z, w)$ é um ponto dual factível, daí:

(ii) $A^{t} y-w+z=c \Rightarrow z=c-A^{t} y+w$.

Usando(ii) em (i):

(iii) $x^{t}\left(c-A^{t} y+w\right)+t^{t} w=c^{t} x-(A x)^{t} y+x^{t} w+t^{t} w$.

Como o ponto é também primal factível, temos:

(iv) $A x=b$ e $x+t=u$.

Sunbstituindo (iv) em (iii):

$c^{t} x-b^{t} y+(u-t)^{t} w+t^{t} w=c^{t} x-b^{t} y+u^{t} w=\gamma$.

Logo, $x^{t} z+t^{t} w=\gamma$. 


\subsection{Método de Pontos Interiores Primal-Dual Preditor- Corretor com Variáveis Canalizadas}

\subsubsection{Construção do Método de Pontos Interiores Preditor-Corretor}

A idéia do método preditor-corretor desenvolvido por Mehrotra [12] consiste em utilizar uma direção que contenha três componentes:

- Direção Afim-Escala (direção preditora ou de Newton).

- Direção de Centragem cujo tamanho é determinado pela perturbação $\mu$.

- Direção de Correção que tenta compensar a aproximação linear do método de Newton dada por $(x+d x)^{t}(z+d z)=d x^{t} d z$.

Ou seja, ao se calcular a direção afim-escala $\mu=0$ verificamos o progresso do método ao longo desta direção. Se o progresso nesta direção for grande, a perturbação $\mu$ é pequena. Caso contrário, é conveniente aumentar o peso da direção de centragem, tal que a perturbação $\mu$ seja grande.

Uma vez que uma segunda direção é calculada, também calculamos a correção não-linear utilizando o mesmo Jacobiano, para que o esforço computacional por iteração não duplique. Para o problema sem canalização, temos os seguintes passos:

Primeira Etapa: Encontrar a direção afim no ponto $(x, y, z)$ :

$$
\left\{\begin{array}{c}
A d \tilde{x}=r_{p} \\
A^{t} d \tilde{y}+d \tilde{z}=r_{d} \\
Z d \tilde{x}+X d \tilde{z}=r_{a}=-X Z e .
\end{array}\right.
$$

Segunda Etapa: Usar o mesmo Jacobiano para calcular a direção de correção $(d \hat{x}, d \hat{y}, d \hat{z})$ no ponto $(\tilde{x}, \tilde{y}, \tilde{z})=(x+d \tilde{x}, y+d \tilde{y}, z+d \tilde{z}):$

$$
\left\{\begin{array}{c}
A d \hat{x}=r_{p}(\tilde{x}, \tilde{y}, \tilde{z})=0 \\
A^{t} d \hat{y}+d \hat{z}=r_{d}(\tilde{x}, \tilde{y}, \tilde{z})=0 \\
Z d \hat{x}+X d \hat{z}=\mu e-(\Delta \tilde{x})(\Delta \tilde{z})=r_{c}, \Delta \tilde{x}=\operatorname{diag}(d \tilde{x}) .
\end{array}\right.
$$


Terceira Etapa: A direção a ser usada é a soma das duas direções, ou seja, $(d x, d y, d z)=(d \tilde{x}, d \tilde{y}, d \tilde{z})+(d \hat{x}, d \hat{y}, d \hat{z})$. Para obter esta direção, somamos os dois sistemas, obtendo:

$$
\left\{\begin{array}{c}
A d x=r_{p} \\
A^{t} d y+d z=r_{d} \\
Z d x+X d z=r_{s}
\end{array}\right.
$$

onde $r_{s}=r_{a}+r_{c}$. Então não precisamos calcular a direção $(d \hat{x}, d \hat{y}, d \hat{z})$ com a segunda etapa, basta utilizarmos $r_{s}$ no lugar de $r_{a}$.

Por analogia, podemos determinar para o problema com canalização, as seguintes etapas:

Primeira Etapa: Encontrar a direção afim no ponto $(x, t, y, w, z)$ :

$$
\left\{\begin{array}{c}
A d \tilde{x}=r_{p} \\
d \tilde{x}+d \tilde{t}=r_{\tilde{u}} \\
A^{t} d \tilde{y}-d \tilde{w}+d \tilde{z}=r_{d} \\
Z d \tilde{x}+X d \tilde{z}=r_{a c}=-X Z e \\
T d \tilde{w}+W d \tilde{t}=r_{a e}=-T W e
\end{array}\right.
$$

Segunda Etapa: Usando o mesmo Jacobiano para calcular a direção de correção $(d \hat{x}, d \hat{t}, d \hat{y}, d \hat{w}, d \hat{z})$ no ponto $(\tilde{x}, \tilde{t}, \tilde{y}, \tilde{w}, \tilde{z})=(x+d \tilde{x}, t+d \tilde{t}, y+d \tilde{y}, w+d \tilde{w}, z+d \tilde{z})$. Por analogia:

$$
\left\{\begin{array}{l}
Z d \hat{x}+X d \hat{z}=\mu e-(\Delta \tilde{x})(\Delta \tilde{z}) ; \operatorname{onde} \Delta \tilde{(} x)=\operatorname{diag}(d \tilde{x}) \\
T d \hat{w}+W d \hat{t}=\mu e-(\Delta \tilde{t})(\Delta \tilde{w}) ; \operatorname{onde} \Delta \tilde{(} t)=\operatorname{diag}(d \tilde{t}) .
\end{array}\right.
$$

Terceira Etapa: A direção a ser usada, a soma das duas direções, é tal que:

$$
\left\{\begin{array}{c}
A d x=r_{p} \\
d x+d t=r_{\tilde{u}} \\
A^{t} d y-d w+d z=r_{d} \\
Z d x+X d z=r_{s c}=r_{a c}+(\mu e-(\Delta \tilde{x})(\Delta \tilde{z})) \\
T d w+W d t=r_{s e}=r_{a e}+(\mu e-(\Delta \tilde{t})(\Delta \tilde{w}))
\end{array}\right.
$$

Antes de construirmos o método, a partir das deduções anteriores, vejamos algumas observações importantes: 
1. Uma vez que estamos utilizando o mesmo Jacobiano, apenas uma decomposição da matriz $\left(A D^{-1} A^{t}\right)$ é calculada. Assim, para calcularmos $d \tilde{y}$ e $d \hat{y}$ é necessário decompor $\left(A D^{-1} A^{t}\right)$ apenas uma vez e resolver dois sistemas com esta decomposição.

2. O cálculo de $\mu$ é função da direção afim. Quanto melhor a direção afim, menor a perturbação e vice-versa. Ou seja:

- $\gamma=\left(x+\alpha_{p} d x\right)^{t}\left(z+\alpha_{d} d z\right)+\left(t+\alpha_{p} d t\right)^{t}\left(w+\alpha_{d} d w\right)$

- $\tilde{\gamma}=\left(x+\tilde{\alpha}_{p} d \tilde{x}\right)^{t}\left(z+\tilde{\alpha}_{d} d \tilde{z}\right)+\left(t+\tilde{\alpha}_{p} d \tilde{t}\right)^{t}\left(w+\tilde{\alpha}_{d} d \tilde{w}\right)$

- $\mu=\sigma\left(\frac{\gamma}{n_{p}}\right)$, onde

$$
\sigma=\left\{\begin{array}{c}
\left(\frac{\tilde{\gamma}}{\gamma}\right)^{3}, \text { se } \gamma \geq 1 \\
\left(\frac{\tilde{\gamma}}{\sqrt{n_{p}}}\right), \text { se } \gamma<1
\end{array}\right.
$$

onde: $\gamma$ é o valor do gap, $n_{p}$ é o número de pares complementares, sendo $\gamma / n_{p}$ o gap médio, $\sigma$ é o patâmetro de centragem e $\mu$ é a perturbação.

3. O método preditor-corretor pode ser obtido com uma pequena modificação em relação ao primal-dual, com pequenos custos computacionais adicionais, mas com ganho no número total de iterações [12].

Após estas observações, vejamos o método para o nosso problema.

\subsubsection{Método de Pontos Interiores Preditor-Corretor}

De acordo com as deduções anteriores, podemos resumir o método preditor-corretor para problemas com variáveis canalizadas:

Dados $\left(x^{0}, t^{0}, w^{0}, z^{0}\right) \geq 0, y^{0}$ livre e $\tau \in(0,1)$.

Para $k=0,1, \ldots$, faça:

$$
\begin{aligned}
r_{p}^{k} & =b-A x^{k} \\
r_{u}^{k} & =u-x^{k}-t^{k} \\
r_{d}^{k} & =c-A^{t} y^{k}+w^{k}-z^{k} \\
r_{a c}^{k} & =-X^{k} Z^{k} e \\
r_{a e}^{k} & =-T^{k} W^{k} e \\
D^{k} & =\left(T^{k}\right)^{-1} W^{k}+\left(X^{k}\right)^{-1} Z^{k}
\end{aligned}
$$




$$
\begin{aligned}
& d y^{k}=\left(A\left(D^{k}\right)^{-1} A^{t}\right)^{-1}\left(r_{p}{ }^{k}+A\left(D^{k}\right)^{-1}\left(r_{d}{ }^{k}+\left(T^{k}\right)^{-1} r_{a e}{ }^{k}-\left(X^{k}\right)^{-1} r_{a c}{ }^{k}-\left(T^{k}\right)^{-1} W^{k} r_{u}{ }^{k}\right)\right) \\
& d x=\left(D^{k}\right)^{-1}\left(A^{t} d y^{k}-r_{d}^{k}-\left(T^{k}\right)^{-1} r_{a e}{ }^{k}+\left(X^{k}\right)^{-1} r_{a c}{ }^{k}+\left(T^{k}\right)^{-1} W^{k} r_{u}{ }^{k}\right) \\
& d t^{k}=r_{u}{ }^{k}-d x^{k} \\
& d z^{k}=\left(X^{k}\right)^{-1}\left(r_{a c}^{k}-Z^{k} d x^{k}\right) \\
& d w^{k}=\left(T^{k}\right)^{-1}\left(r_{a e}{ }^{k}-W^{k} d t^{k}\right) \\
& \rho_{p}^{k}=\min \left\{\frac{-1}{\min _{i}\left(\frac{d x^{k}}{x_{i}^{k}}\right)}, \frac{-1}{\min _{i}\left(\frac{d t^{k}}{t_{i}^{k}}\right)}\right\} \\
& \rho_{d}^{k}=\min \left\{\frac{-1}{\min _{i}\left(\frac{d w^{k}}{w_{i}^{k}}\right)}, \frac{-1}{\min _{i}\left(\frac{d z^{k}}{z_{i}^{k}}\right)}\right\} \\
& \alpha_{p}^{k}=\min \left(1, \tau \rho_{p}^{k}\right) \\
& \alpha_{d}^{k}=\min \left(1, \tau \rho_{d}^{k}\right) \\
& \gamma^{k}=\left(x^{k}+\alpha_{p}^{k} d x^{k}\right)^{t}\left(z^{k}+\alpha_{d}^{k} d z^{k}\right)+\left(t^{k}+\alpha_{p}^{k} d t^{k}\right)^{t}\left(w^{k}+\alpha_{d}^{k} d w^{k}\right) \\
& \tilde{\gamma}^{k}=\left(x^{k}+\tilde{\alpha}_{p}^{k} d \tilde{x}^{k}\right)^{t}\left(z^{k}+\tilde{\alpha}_{d}^{k} d \tilde{z}^{k}\right)+\left(t^{k}+\tilde{\alpha}_{p}^{k} d \tilde{t}^{k}\right)\left(w^{k}+\tilde{\alpha}_{d}^{k} d \tilde{w}^{k}\right) \\
& \mu^{k}=\sigma \frac{\gamma^{k}}{n} \\
& r_{s c}^{k}=r_{a c}^{k}+\mu^{k} e-\left(\Delta \tilde{x}^{k}\right)\left(\Delta \tilde{z}^{k}\right) e \\
& r_{s e}^{k}=r_{a e}^{k}+\mu^{k} e-\left(\Delta \tilde{t}^{k}\right)\left(\Delta \tilde{w}^{k}\right) e \\
& d y^{k}=\left(A\left(D^{k}\right)^{-1} A^{t}\right)^{-1}\left(r_{p}{ }^{k}+A\left(D^{k}\right)^{-1}\left(r_{d}{ }^{k}+\left(T^{k}\right)^{-1} r_{s e}{ }^{k}-\left(X^{k}\right)^{-1} r_{s c}{ }^{k}-\left(T^{k}\right)^{-1} W^{k} r_{u}{ }^{k}\right)\right) \\
& d x=\left(D^{k}\right)^{-1}\left(A^{t} d y^{k}-r_{d}{ }^{k}-\left(T^{k}\right)^{-1} r_{s e}{ }^{k}+\left(X^{k}\right)^{-1} r_{s c}{ }^{k}+\left(T^{k}\right)^{-1} W^{k} r_{u}{ }^{k}\right) \\
& d t^{k}=r_{u}^{k}-d x^{k} \\
& d z^{k}=\left(X^{k}\right)^{-1}\left(r_{s c}^{k}-Z^{k} d x^{k}\right) \\
& d w^{k}=\left(T^{k}\right)^{-1}\left(r_{s e}{ }^{k}-W^{k} d t^{k}\right) \\
& x^{k+1}=x^{k}+\alpha^{p} d x^{k} \\
& t^{k+1}=t^{k}+\alpha^{p} d t^{k} \\
& y^{k+1}=y^{k}+\alpha^{d} d y^{k} \\
& z^{k+1}=z^{k}+\alpha^{d} d z^{k} \\
& w^{k+1}=w^{k}+\alpha^{d} d w^{k}
\end{aligned}
$$

Até convergir. 


\subsection{Extensão do Método de Pontos Interiores para o Problema de Programação Quadrática Convexa}

A motivação para se abordar o problema de programação quadrática convexa, neste trabalho, é que o mesmo será utilizado para a resolução do problema de fluxo de potência ótimo.

Neste caso, vamos minimizar uma função objetivo quadrática convexa, sujeita a restrições lineares, cujo problema primal na forma padrão é dado por:

$$
\begin{array}{cc}
\min & c^{t} x+\frac{1}{2} x^{t} Q x \\
\text { sa } & A x=b \\
& x \geq 0 .
\end{array}
$$

Onde $Q$ é uma matriz simétrica definida positiva de dimensão $n x n$. Para o Problema de fluxo de potência ótimo $Q$ é uma matriz diagonal, hipótese que assumiremos a partir de agora.

A partir da formulação primal dada em (3.16), podemos escrever o problema dual como segue:

$$
\begin{array}{cc}
\max & b^{t} y-\frac{1}{2} x^{t} Q x \\
\text { sa } & A^{t} y-Q x+z=c \\
& x, z \geq 0, y \text { livre. }
\end{array}
$$

\subsubsection{Dedução do Método}

De posse das formulações primal e dual do problema iremos obter as condições de otimalidade que são dadas por:

$$
\begin{gathered}
\text { factibilidade primal: }\left\{\begin{array}{c}
b-A x=0 \\
(x, t) \geq 0,
\end{array}\right. \\
\text { factibilidade dual: }\left\{\begin{array}{c}
c-A^{t} y+Q x-z=0 \\
(x, z) \geq 0 \\
y \text { livre, }
\end{array}\right.
\end{gathered}
$$


condições de complementaridade: $\{X Z e=0$.

Aplicando o método de Newton às condições de otimalidade, obtemos:

$$
\left[\begin{array}{ccc}
A & 0 & 0 \\
Q & -A^{t} & -I \\
Z & 0 & X
\end{array}\right]\left[\begin{array}{l}
d x \\
d y \\
d z
\end{array}\right]=\left[\begin{array}{c}
r_{p} \\
r_{d} \\
r_{c}
\end{array}\right]
$$

onde:

$$
\left\{\begin{array}{rrr}
r_{p}= & b-A x \\
r_{d}= & c-A^{t} y+Q x-z \\
r_{c}= & \mu e-X Z e .
\end{array}\right.
$$

Reescrevendo o sistema linear, temos:

$$
\left\{\begin{array}{c}
A d x=r_{p} \\
A^{t} d y-Q d w+d z=r_{d} \\
Z d x+X d z=r_{c}
\end{array}\right.
$$

Considerando que $Z$ e $X$ são matrizes diagonais com elementos positivos, e portanto inversíveis, então, com a última equação de (3.18), chegamos em:

$$
\left\{d z=X^{-1}\left(r_{c}-Z d x\right)\right.
$$

Agora, aplicando (3.19) na segunda equação de (3.18):

$$
A^{t} d y-Q d x+X^{-1} Z d x=r_{d}-X^{-1} r_{c}=r_{M}
$$

Assim, nosso sistema é simplificado para:

$$
\left\{\begin{array}{c}
A d x=r_{p} \\
A^{t} d y-D d x=r_{M} .
\end{array}\right.
$$

A matriz $D=\left(Q+X^{-1} Z\right)$ pode ser facilmente invertida, pois ela é a soma de matrizes diagonais definidas positivas. Então, podemos eliminar $d x$ da segunda equação de (3.21), e substituindo $r_{M}$, temos:

$$
d x=D^{-1}\left(A^{t} d y-r_{d}+X^{-1} r_{c}\right)
$$


Substituindo $d x$ na primeira equação de (3.21), temos:

$$
\left(A D^{-1} A^{t}\right) d y-r_{p}=A D^{-1}\left(r_{d}-X^{-1} r_{c}\right)
$$

Cabe destacar que a inversão de $A D^{-1} A^{t}$ em (3.23) envolve a maior parte do esforço computacional do nosso método. Mas, essa matriz é simétrica e definida positiva e, na prática, utilizamos a decomposição de Cholesky para resolver o sistema linear [6].

Podemos verificar que o esforço computacional por iteração deste método é praticamente o mesmo que o do método para programação linear.

\subsubsection{Critério de Convergência e Valor do Gap}

O critério de convergência é dado por:

$$
\max \left\{\begin{array}{c}
\frac{\|b-A x\|}{1+\|b\|} \\
\frac{\left\|c-A^{t} y+Q x-z\right\|}{1+\|c\|} \\
\frac{c^{t} x-b^{t} y+x^{t} Q x}{1+\left\|c^{t} x-b^{t} y+x^{t} Q x\right\|}
\end{array}\right\} \leq \varepsilon,
$$

onde $\varepsilon$ é a tolerância estabelecida.

Com o propósito de simplificação dos cálculos a serem efetuados pelo método apresentado nesta seção, vamos provar a equivalência de uma fórmula mais simples para o valor do gap. Ou seja, vamos demonstrar que o gap dado por $\gamma=c^{t} x-b^{t} y+x^{t} Q x$ equivale a $\gamma=x^{t} z$, para todo ponto factível.

Seja

(i) $x^{t} z$;

Temos que $(x, z)$ é um ponto dual factível, daí:

(ii) $A^{t} y-Q x+z=c \Rightarrow z=c-A^{t} y+Q x$.

Usando(ii) em (i):

(iii) $x^{t}\left(c-A^{t} y+Q x\right)=c^{t} x-(A x)^{t} y+x^{t} Q x$.

Como o ponto é também primal factível, temos:

(iv) $A x=b$.

Sunbstituindo (iv) em (iii):

$c^{t} x-b^{t} y+x^{t} Q x=\gamma$.

Logo, $x^{t} z=\gamma$. 


\section{Capítulo 4}

\section{Aplicação de Métodos de Pontos}

\section{Interiores no Problema de Fluxo de}

\section{Potência}

A proposta deste capítulo é desenvolver métodos de pontos interiores que sejam adequados aos problemas de fluxo de potência ótimo utilizando os modelos de fluxos em rede e o princípio do mínimo esforço, apresentados no Capítulo 2. Ou seja, o Capítulo 3 forneceu deduções para métodos de propósito geral, aqui, tiramos proveito das particularidades dos problemas para criar métodos mais eficiente que os de propósito geral.

\subsection{Métodos de Pontos Interiores Aplicados ao Fluxo de Potência Ótimo}

\subsubsection{Método de Pontos Interiores Primal-Dual}

Já vimos, no Capítulo 2 Seção 2.2, que o problema de fluxo de potência ótimo, utilizando o modelo de fluxos em rede, é dado por:

$$
\begin{aligned}
& \min \quad \alpha f^{t} R f+\beta\left(p^{t} H p+c^{t} p\right) \\
& \text { sa } \quad A f=p-l, T f=0 \\
& f^{\text {min }} \leq f \leq f^{\max }, p^{\min } \leq p \leq p^{\max }
\end{aligned}
$$


Para simplificar o desenvolvimento do método, faremos as seguintes alterações no modelo:

- Mudança de variáveis $\tilde{f}=f-f^{m i n}$ e $\tilde{p}=p-p^{m i n}$.

- As ponderações $\alpha$ e $\beta$ terão valor unitário.

Após estas alterações, temos a formulação dada por:

$$
\begin{array}{cc}
\min & \tilde{f}^{t} \tilde{R} \tilde{f}+c_{f}^{t} \tilde{f}+\tilde{p}^{t} H \tilde{p}+c_{p}{ }^{t} \tilde{p} \\
s a & A \tilde{f}-\tilde{p}=\tilde{l}_{i}, T \tilde{f}=\tilde{l}_{v} \\
& 0 \leq \tilde{f} \leq \tilde{f}^{\text {max }}, 0 \leq \tilde{p} \leq \tilde{p}^{\text {max }},
\end{array}
$$

onde $c_{f}=2 \tilde{R} f^{\text {min }}, c_{p}=2 H p^{\text {min }}+c, \tilde{l}_{i}=p^{\text {min }}-l-A f^{\text {min }}$ e $\tilde{l}_{v}=-T f^{\text {min }}$.

Introduzindo as variáveis de folga das restrições de capacidade e eliminando os "tils" para maior clareza do texto, temos (4.2).

$$
\begin{array}{cc}
\min & f^{t} R f+c_{f}^{t} f+p^{t} H p+c_{p}{ }^{t} p \\
\text { sa } & A f-p=l_{i}, T f=l_{v} \\
& 0 \leq f \leq f^{\max }, 0 \leq p \leq p^{\max } \\
& \left(f, s_{f}\right) \geq 0,\left(p, s_{p}\right) \geq 0 .
\end{array}
$$

Ao problema primal dado por (4.2), associamos o problema dual formulado em (4.3), já com as variáveis de folga introduzidas.

$$
\begin{array}{cc}
\max \quad l^{t} y-\left(f^{\max }\right)^{t} w_{f}-f^{t} R f-\left(p^{\max }\right)^{t} w_{p}-p^{t} H p \\
s a \\
C^{t} y+z_{f}-w_{f}-R f=c_{f} \\
-y(p)+z_{p}-w_{p}-H p=c_{p} \\
\left(z_{p}, w_{p}\right) \geq 0,\left(z_{f}, w_{f}\right) \geq 0,
\end{array}
$$

onde $C=\left(\begin{array}{c}A \\ T\end{array}\right), l=\left(\begin{array}{c}l_{i} \\ l_{v}\end{array}\right)$ e $y(p)$ representa os elementos da variável dual $y$ correspondentes às barras de geração.

As condições de otimalidade para os problemas (4.2) e (4.3) são dadas pela factibilidade primal e dual, e pelas condições de complementaridade: 
factibilidade primal: $\left\{\begin{array}{c}A f-p=l_{i} \\ T f=l_{v} \\ f+s_{f}=f^{\text {max }} \\ p+s_{p}=p^{\text {max }} \\ \left(f, s_{f}\right) \geq 0\left(p, s_{p}\right) \geq 0, \\ C^{t} y+z_{f}-w_{f}-R f=c_{f} \\ -y(p)+z_{p}-w_{p}-H p=c_{p} \\ \left(z_{p}, w_{p}\right) \geq 0 \\ \left(z_{f}, w_{f}\right) \geq 0\end{array}\right.$

condições de complementaridade: $\left\{\begin{array}{c}F Z_{f} e=0 \\ P Z_{p} e=0 \\ S_{f} W_{f} e=0 \\ S_{p} W_{p} e=0 .\end{array}\right.$

Aplicando o método de Newton nas condições de otimalidade, obtemos o método de pontos interiores primal-dual para o fluxo de potência ótimo, como a seguir:

$$
\left[\begin{array}{cccccccccc}
A & -I & 0 & 0 & 0 & 0 & 0 & 0 & 0 & 0 \\
T & 0 & 0 & 0 & 0 & 0 & 0 & 0 & 0 & 0 \\
I & 0 & I & 0 & 0 & 0 & 0 & 0 & 0 & 0 \\
0 & I & 0 & I & 0 & 0 & 0 & 0 & 0 & 0 \\
-R & 0 & 0 & 0 & C^{t} & 0 & I & -I & 0 & 0 \\
0 & -H & 0 & 0 & 0 & -I & 0 & 0 & I & -I \\
Z_{f} & 0 & 0 & 0 & 0 & 0 & F & 0 & 0 & 0 \\
0 & Z_{p} & 0 & 0 & 0 & 0 & 0 & 0 & P & 0 \\
0 & 0 & W_{f} & 0 & 0 & 0 & 0 & S_{f} & 0 & 0 \\
0 & 0 & 0 & W_{p} & 0 & 0 & 0 & 0 & 0 & S_{p}
\end{array}\right]\left[\begin{array}{c}
d f \\
d p \\
d s_{f} \\
d s_{p} \\
d y \\
d y_{p} \\
d z_{f} \\
d w_{f} \\
d z_{p} \\
d w_{p}
\end{array}\right]=\left[\begin{array}{c}
r_{i} \\
r_{v} \\
r_{f} \\
r_{p} \\
r_{y} \\
r_{g} \\
r_{z f} \\
r_{z p} \\
r_{w f} \\
r_{w p}
\end{array}\right] .
$$


Ou seja, temos o seguinte sistema:

$$
\left\{\begin{array}{c}
A d f-d p=r_{i} \\
T d f=r_{v} \\
d f+d s_{f}=r_{f} \\
d p+d s_{p}=r_{p} \\
C^{t} d y+d z_{f}-d w_{f}-R d f=r_{y} \\
-d y(p)+d z_{p}-d w_{p}-H d p=r_{g} \\
Z_{f} d f+F d z_{f}=r_{z f} \\
Z_{p} d p+P d z_{p}=r_{z p} \\
W_{f} d s_{f}+S_{f} d w_{f}=r_{w f} \\
W_{p} d s_{p}+S_{p} d w_{p}=r_{w p} .
\end{array}\right.
$$

Onde os resíduos são:

$$
\left\{\begin{array}{c}
r_{i}=l_{i}+p-A f \\
r_{v}=l_{v}-T f \\
r_{f}=f^{\max }-f-s_{f} \\
r_{p}=p^{\max }-p-s_{p} \\
r_{y}=c_{f}-C^{t} y-z_{f}+w_{f}+R f \\
r_{g}=c_{p}+y(p)-z_{p}+w_{p}+H p \\
r_{z f}=\mu e-F Z_{f} e \\
r_{z p}=\mu e-P Z_{p} e \\
r_{w f}=\mu e-S_{f} W_{f} e \\
r_{w p}=\mu e-S_{p} W_{p} e .
\end{array}\right.
$$

O sistema linear (4.4) pode ser simplificado através da eliminação de variáveis sem modificar sua estrutura esparsa.

Substituindo as variáveis de folga, temos:

$$
\begin{aligned}
d s_{f} & =r_{f}-d f \\
d s_{p} & =r_{p}-d p \\
d z_{f} & =F^{-1}\left(r_{z f}-Z_{f} d_{f}\right) \\
d z_{p} & =P^{-1}\left(r_{z p}-Z_{p} d_{p}\right)
\end{aligned}
$$




$$
\begin{aligned}
& d w_{f}=S_{f}^{-1}\left(r_{w f}-W_{f} d s_{f}\right) ; \\
& d w_{p}=S_{p}^{-1}\left(r_{w p}-W_{p} d s_{p}\right) .
\end{aligned}
$$

Com essas substituições, o sistema (4.4) se reduz a:

$$
\left\{\begin{array}{c}
A d f-d p=r_{i} \\
T d f=r_{v} \\
C^{t} d y-D_{f} d f=r_{a} \\
-d y(p)-D_{p} d p=r_{b},
\end{array}\right.
$$

onde $D_{f}=F^{-1} Z_{f}+S_{f}^{-1} W_{f}+R, D_{p}=P^{-1} Z_{p}+S_{p}^{-1} W_{p}+H, r_{a}=r_{y}-F^{-1} r_{z f}+S_{f}^{-1} r_{w f}-$ $S_{f}^{-1} W_{f} r_{f}$ e $r_{b}=r_{g}-P^{-1} r_{z p}+S_{p}^{-1} r_{w p}-S_{p}^{-1} W_{p} r_{p}$.

Podemos ver que somente inversas e matrizes diagonais são envolvidas. Eliminando as variáveis de geração e fluxo de potência em (4.5), temos:

$$
\begin{aligned}
& d f=-D_{f}^{-1}\left(r_{a}-C^{t} d y\right) ; \\
& d p=-D_{p}^{-1}\left(r_{b}+d y(p)\right) .
\end{aligned}
$$

Utilizando estes valores nas outras equações de (4.5):

$$
\begin{gathered}
A D_{f}{ }^{-1} C^{t} d y+D_{p}^{-1} d y(p)=r_{i}+A D_{f}^{-1} r_{a}-D_{p}^{-1} r_{b} \\
T D_{f}^{-1} C^{t} d y=r_{v}+T D_{f}^{-1} r_{a} .
\end{gathered}
$$

Fazendo $C=\left(\begin{array}{c}A \\ T\end{array}\right), D=\left(\begin{array}{c}-D_{p}^{-1} \\ 0\end{array}\right), d y(p)=D d y$ e $\tilde{r}=\left(\begin{array}{c}r_{i} \\ r_{v}\end{array}\right)$, e utilizando conjuntamente as equações de(4.6), obtemos:

$$
\left(C D_{f}^{-1} C^{t}+D\right) d y=r
$$

onde $r=\tilde{r}+C D_{f}^{-1} r_{a}-D r_{b}$.

Novamente somente matrizes diagonais são invertidas.

O fato de a matriz $C$ ter dimensão $n+1 x n$ é bastante relevante. Esta característica pode ser utilizada para reduzir o esforço computacional por iteração dos métodos de pontos interiores, assim, se formarmos a matriz não singular $\tilde{C}=\left[\begin{array}{ll}C & e_{j}\end{array}\right]$, onde $e_{j}$ representa um 
vetor canônico (vetor formado pelas colunas da matriz identidade), onde $j$ é um índice referente a uma barra de geração, o sistema (4.7) pode ser reescrito na seguinte forma:

$$
\left(\tilde{C} \tilde{D}_{f}^{-1} \tilde{C}^{t}+\tilde{D}\right) d y=r
$$

onde $\tilde{D}_{f}^{-1}$ incorpora o elemento diagonal retirado de $D$ para formar $\tilde{D}$. A resolução de (4.8) se dá em duas etapas. Na primeira, um sistema linear contendo apenas a matriz $\tilde{C} \tilde{D}_{f}^{-1} \tilde{C}^{t}$ é resolvido. Vale observar que como $\tilde{C}$ é quadrada, a resolução do sistema com a matriz $\tilde{C} \tilde{D}_{f}^{-1} \tilde{C}^{t}$ fica muito barata, pois apenas a matriz diagonal $\tilde{D}_{f}$ varia a cada iteração. Então, apenas uma decomposição de $\tilde{C}$ é necessária e pode ser realizada antes da aplicação do método iterativo.

Na segunda etapa, a fórmula de Sherman-Morrison-Woodbury [6] é aplicada para a obtenção de $d y$ :

$$
\begin{aligned}
W & =\tilde{C}^{-1} E \\
Z & =W^{t} \tilde{D}_{f} W \\
v & =\left(\tilde{C} \tilde{D}_{f}^{-1} \tilde{C}^{t}\right)^{-1} r \\
d y & =v-\left(\tilde{C} \tilde{D}_{f}^{-1} \tilde{C}^{t}\right)^{-1} E\left(\tilde{D}^{-1}+Z\right)^{-1} E^{t} v,
\end{aligned}
$$

onde $E$ é formada por vetores canônicos correspondentes aos elementos diagonais não nulos de $\tilde{D}$. Consequentemente, $W$ é fixa para uma determinada rede e pode ser calculada antes da aplicação do método iterativo. Podemos observar que a multiplicação de $E$ ou $E^{t}$ por um vetor não envolve operações de ponto flutuante, uma vez que as colunas de $E$ são colunas da matriz identidade.

Podemos, agora, formalizar o método de pontos interiores primal-dual para o problema de fluxo de potência ótimo DC como segue:

Dados $\left(f^{0}, p^{0}, s_{f}^{0}, s_{p}^{0}, z_{f}^{0}, w_{f}^{0}, z_{p}^{0}, w_{p}^{0}\right) \geq 0, y^{0}$ livre e $\sigma \in(0,1)$.

Sejam $x=\left(f, p, s_{f}, s_{p}\right), t=\left(z_{f}, w_{f}, z_{p}, w_{p}\right)$ e $n_{p}$ a dimensão do vetor $x$.

Para $k=0,1,2, \ldots$, faça:

$\mu^{k}=\sigma \frac{\gamma^{k}}{n_{p}}$.

Calcule os resíduos $r_{i}^{k}, r_{v}^{k}, r_{f}^{k}, r_{p}^{k}, r_{y}^{k}, r_{g}^{k}, r_{z f}^{k}, r_{z p}^{k}, r_{w f}^{k}, r_{w p}^{k}, r_{a}^{k}$ e $r_{b}^{k}$.

$$
D_{f}^{k}=\left(F^{k}\right)^{-1} Z_{f}^{k}+\left(S_{f}^{k}\right)^{-1} W_{f}^{k}+R
$$




$$
\begin{aligned}
& r^{k}=\left(\begin{array}{c}
r_{i}{ }^{k} \\
r_{v}{ }^{k}
\end{array}\right)+C\left(D_{f}^{k}\right)^{-1} r_{a}^{k}-D r_{b}^{k} \\
& Z^{k}=W^{t} \tilde{D}_{f}^{k} W \\
& v^{k}=\left(\tilde{C}^{-t}\left(\tilde{D}_{f}^{k}\right)^{-1} \tilde{C}^{t}\right)^{-1} r^{k} \\
& d y^{k}=v^{k}-\left(\tilde{C}^{-t}\left(\tilde{D}_{f}^{k}\right)^{-1} \tilde{C}^{t}\right)^{-1} E\left(\tilde{D}^{-1}+Z^{k}\right)^{-1} E^{t} v^{k} \\
& d f^{k}=-\left(D_{f}^{k}\right)^{-1}\left(r_{a}^{k}-C^{t} d y^{k}\right) \\
& d y(p)^{k}=\left(\begin{array}{c}
-\left(D_{p}^{k}\right)^{-1} \\
0
\end{array}\right) d y^{k} \\
& D_{p}^{k}=\left(P^{k}\right)^{-1} Z_{p}^{k}+\left(S_{p}^{k}\right)^{-1} W_{p}^{k}+H \\
& d p^{k}=-\left(D_{p}^{k}\right)^{-1}\left(r_{b}^{k}+d y(p)^{k}\right) \\
& d s_{f}^{k}=r_{f}^{k}-d f^{k} \\
& d s_{p}^{k}=r_{2}^{k}-d p^{k} \\
& d z_{f}^{k}=\left(Z^{k}\right)^{-1}\left(r_{z f}^{k}-Z_{f}^{k} d_{f}^{k}\right) \\
& d z_{p}^{k}=\left(P^{k}\right)^{-1}\left(r_{z p}^{k}-Z_{p}^{k} d_{p}^{k}\right) \\
& d w_{f}^{k}=\left(S_{f}^{k}\right)^{-1}\left(r_{w f}^{k}-W_{f}^{k} d s_{f}^{k}\right) \\
& d w_{p}^{k}=\left(S_{p}^{k}\right)^{-1}\left(r_{w p}^{k}-W_{p}^{k} d s_{p}^{k}\right) \\
& d x^{k}=\left(d f^{k}, d p^{k}, d s f^{k}, d s_{p}^{k}\right) \\
& d t^{k}=\left(d z_{f}^{k}, d w_{f}^{k}, d z_{p}^{k}, d w_{p}^{k}\right) \\
& \rho_{p}^{k}=\frac{-1}{\min _{i}\left(\frac{d x^{k}}{x_{i}^{k}}\right)} \\
& \rho_{d}^{k}=\frac{-1}{\min _{i}\left(\frac{d t^{k}}{t_{i}^{k}}\right)} \\
& \alpha_{p}^{k}=\min \left(1, \tau \rho_{p}^{k}\right) \\
& \alpha_{d}^{k}=\min \left(1, \tau \rho_{d}^{k}\right) \\
& x^{k+1}=x^{k}+\alpha^{k} d x^{k} \\
& y^{k+1}=y^{k}+\alpha^{k} d y^{k} \\
& t^{k+1}=t^{k}+\alpha^{k} d t^{k}
\end{aligned}
$$

Até convergir.

Este método foi implementado em [20, 22], obtendo excelente desempenho computacional. 


\subsubsection{Método de Pontos Interiores Preditor-Corretor}

Como já vimos, a idéia do método preditor-corretor é utilizar uma direção que contenha três componentes:

- Direção Afim-Escala.

- Direção de Centragem.

- Direção de Correção.

Então, temos de resolver dois sistemas lineares para determinar as direções.

Primeiramente, é calculada a direção afim $(d \tilde{x}, d \tilde{y}, d \tilde{t})$, onde $d \tilde{x}=\left(d \tilde{f}, d \tilde{p}, d \tilde{s_{f}}, d \tilde{s_{p}}\right)$ e $d \tilde{t}=\left(d \tilde{z_{f}}, d \tilde{w}_{f}, d \tilde{z_{p}}, d \tilde{w}_{p}\right)$, resolvendo o sistema linear abaixo:

$$
\left\{\begin{array}{c}
A d \tilde{f}-d \tilde{p}=r_{i} \\
T d \tilde{f}=r_{v} \\
d \tilde{f}+d \tilde{s_{f}}=r_{f} \\
d \tilde{p}+d \tilde{s_{p}}=r_{p} \\
C^{t} d \tilde{y}+d \tilde{z_{f}}-d \tilde{w_{f}}-R d \tilde{f}=r_{y} \\
-d y \tilde{y}(p)+d \tilde{z_{p}}-d \tilde{w_{p}}-H d \tilde{p}=r_{g} \\
Z_{f} d \tilde{f}+F d \tilde{z_{f}}=r_{z f}^{\tilde{f}} \\
Z_{p} d \tilde{p}+P d \tilde{z_{p}}=r_{z p}^{\tilde{z}} \\
W_{f} d \tilde{s_{f}}+S_{f} d \tilde{w_{f}}=r_{w f}^{\tilde{y}} \\
W_{p} d \tilde{s_{p}}+S_{p} d \tilde{w_{p}}=r_{w p}
\end{array}\right.
$$


Onde os resíduos são:

$$
\left\{\begin{array}{c}
r_{i}=l_{i}+p-A f \\
r_{v}=l_{v}-T f \\
r_{f}=f^{\max }-f-s_{f} \\
r_{p}=p^{\max }-p-s_{p} \\
r_{y}=c_{f}-C^{t} y-z_{f}+w_{f}+R f \\
r_{g}=c_{p}+y(p)-z_{p}+w_{p}+H p \\
r_{z f}^{\tilde{y}}=-F Z_{f} e \\
r_{z p}^{\tilde{z}}=-P Z_{p} e \\
r_{w f}^{\sim}=-S_{f} W_{f} e \\
r_{w p}^{\sim}=-S_{p} W_{p} e .
\end{array}\right.
$$

Em seguida, a direção desejada é obtida resolvendo o seguinte sistema linear:

$$
\left\{\begin{array}{c}
A d f-d p=r_{i} \\
T d f=r_{v} \\
d f+d s_{f}=r_{f} \\
d p+d s_{p}=r_{p} \\
C^{t} d y+d z_{f}-d w_{f}-R d f=r_{y} \\
-d y(p)+d z_{p}-d w_{p}-H d p=r_{g} \\
Z_{f} d f+F d z_{f}=r_{z f} \\
Z_{p} d p+P d z_{p}=r_{z p} \\
W_{f} d s_{f}+S_{f} d w_{f}=r_{w f} \\
W_{p} d s_{p}+S_{p} d w_{p}=r_{w p} .
\end{array}\right.
$$

Onde os novos resíduos são:

$$
\left\{\begin{array}{c}
r_{z f}=\mu e-F Z_{f} e-\Delta \tilde{F} \Delta \tilde{Z}_{f} \\
r_{z p}=\mu e-P Z_{p} e-\Delta \tilde{P} \Delta \tilde{Z}_{p} \\
r_{w f}=\mu e-S_{f} W_{f} e-\Delta \tilde{S}_{f} \Delta \tilde{W}_{f} \\
r_{w p}=\mu e-S_{p} W_{p} e-\Delta \tilde{S}_{p} \Delta \tilde{W}_{p}
\end{array}\right.
$$

O cálculo de $\mu$ é função da direção afim. Quanto melhor a direção afim, menor a perturbação e vice-versa. Ou seja:

- $\gamma=x^{t} t$ 
- $\tilde{\gamma}=\left(x+\tilde{\alpha_{p}} d \tilde{x}\right)^{t}\left(t+\tilde{\alpha_{d}} d \tilde{t}\right)$;

- $\mu=\sigma\left(\frac{\gamma}{n_{p}}\right)$, onde

$$
\sigma=\left\{\begin{array}{c}
\left(\frac{\tilde{\gamma}}{\gamma}\right)^{3}, \text { se } \gamma \geq 1 \\
\left(\frac{\tilde{\gamma}}{\sqrt{n_{p}}}\right), \text { se } \gamma<1 .
\end{array}\right.
$$

De acordo com as deduções anteriores, podemos resumir o método de pontos interiores preditor-corretor para o problema de fluxo de potência ótimo DC como segue:

Dados $\left(f^{0}, p^{0}, s_{f}^{0}, s_{p}^{0}, z_{f}^{0}, w_{f}^{0}, z_{p}^{0}, w_{p}^{0}\right) 0, y^{0}$ livre e $\tau \in(0,1)$.

Sejam $x=\left(f, p, s_{f}, s_{p}\right), t=\left(z_{f}, w_{f}, z_{p}, w_{p}\right)$ e $n_{p}=\operatorname{dim}(x)$.

Para $k=0,1,2, \ldots$, faça:

Calcule os resíduos $r_{i}^{k}, r_{v}^{k}, r_{f}^{k}, r_{p}^{k}, r_{y}^{k}, r_{g}^{k}, r_{z f}^{k}, \tilde{r}_{z p}^{k}, r_{w f}^{*}, r_{w p}^{*}, r_{a}^{k}$ e $r_{b}^{k}$.

$$
\begin{aligned}
& D_{f}^{k}=\left(F^{k}\right)^{-1} Z_{f}^{k}+\left(S_{f}^{k}\right)^{-1} W_{f}^{k}+R \\
& r^{k}=\left(\begin{array}{c}
r_{i}{ }^{k} \\
r_{v}{ }^{k}
\end{array}\right)+C\left(D_{f}^{k}\right)^{-1} r_{a}^{k}-D r_{b}^{k} \\
& Z^{k}=W^{t} \tilde{D}_{f}^{k} W \\
& v^{k}=\left(\tilde{C}^{-t}\left(\tilde{D}_{f}^{k}\right)^{-1} \tilde{C}^{t}\right)^{-1} r^{k} \\
& d \tilde{y}^{k}=v^{k}-\left(\tilde{C}^{-t}\left(\tilde{D}_{f}^{k}\right)^{-1} \tilde{C}^{t}\right)^{-1} E\left(\tilde{D}^{-1}+Z^{k}\right)^{-1} E^{t} v^{k} \\
& d \tilde{f}^{k}=-\left(D_{f}^{k}\right)^{-1}\left(r_{a}^{k}-C^{t} d \tilde{y}^{k}\right) \\
& d y(p)^{k}=\left(\begin{array}{c}
-\left(D_{p}^{k}\right)^{-1} \\
0
\end{array}\right) d \tilde{y}^{k} \\
& D_{p}^{k}=\left(P^{k}\right)^{-1} Z_{p}^{k}+\left(S_{p}^{k}\right)^{-1} W_{p}^{k}+H \\
& \left.d \tilde{p}^{k}=-\left(D_{p}^{k}\right)^{-1}\left(r_{b}^{k}+d y \tilde{(p}\right)^{k}\right) \\
& d{\tilde{s_{f}}}^{k}=r_{f}^{k}-d \tilde{f}^{k} \\
& d{\tilde{s_{p}}}^{k}=r_{2}^{k}-d \tilde{p}^{k} \\
& d{\tilde{z_{f}}}^{k}=\left(Z^{k}\right)^{-1}\left(r \tilde{z} f^{k}-Z_{f}^{k} d \tilde{f}^{k}\right) \\
& d{\tilde{z_{p}}}^{k}=\left(P^{k}\right)^{-1}\left(\tilde{r}_{z p}{ }^{k}-Z_{p}^{k} d \tilde{p}^{k}\right) \\
& d \tilde{w}_{f}^{k}=\left(S_{f}^{k}\right)^{-1}\left(\tilde{r}_{w f}^{k}-W_{f}^{k} d{\tilde{s_{f}}}^{k}\right) \\
& d \tilde{w}_{p}{ }^{k}=\left(S_{p}^{k}\right)^{-1}\left(\tilde{r}_{w p}{ }^{k}-W_{p}^{k} d{\tilde{s_{p}}}^{k}\right) \\
& d \tilde{x}^{k}=\left(d \tilde{f}^{k}, d \tilde{p}^{k}, d{\tilde{s_{f}}}^{k}, d{\tilde{s_{p}}}^{k}\right)
\end{aligned}
$$




$$
\begin{aligned}
d \tilde{t}^{k} & =\left(d{\tilde{z_{f}}}^{k}, d{\tilde{w_{f}}}^{k}, d{\tilde{z_{p}}}^{k}, d \tilde{w}_{p}^{k}\right) \\
{\tilde{\rho_{p}}}^{k} & =\frac{-1}{\min _{i}\left(\frac{d \tilde{x}^{k}}{x_{i}^{k}}\right)} \\
{\tilde{\rho_{d}}}^{k} & =\frac{-1}{\min _{i}\left(\frac{d \tilde{t}^{k}}{t_{i}^{k}}\right)} \\
{\tilde{\alpha_{p}}}^{k} & =\min \left(1, \tau{\tilde{\rho_{p}}}^{k}\right) \\
{\tilde{\alpha_{d}}}^{k} & =\min \left(1, \tau{\tilde{\rho_{d}}}^{k}\right) \\
\tilde{\gamma}^{k} & =\left(x^{k}+{\tilde{\alpha_{p}}}^{k} d \tilde{x}^{k}\right)^{t}\left(t^{k}+{\tilde{\alpha_{d}}}^{k} d \tilde{t}^{k}\right)
\end{aligned}
$$

Calcule $\sigma^{k}$ e $\mu^{k}$.

Calcule os resíduos $r_{z f}^{k}, r_{z p}^{k}, r_{w f}^{k}$ e $r_{w p}^{k}$.

$$
\begin{aligned}
r^{k} & =\left(\begin{array}{c}
r_{i}^{k} \\
r_{v}^{k}
\end{array}\right)+C\left(D_{f}^{k}\right)^{-1} r_{a}^{k}-D r_{b}^{k} \\
Z^{k} & =W^{t} \tilde{D}_{f}^{k} W \\
v^{k} & =\left(\tilde{C}^{-t}\left(\tilde{D}_{f}^{k}\right)^{-1} \tilde{C}^{t}\right)^{-1} r^{k} \\
d y^{k} & =v^{k}-\left(\tilde{C}^{-t}\left(\tilde{D}_{f}^{k}\right)^{-1} \tilde{C}^{t}\right)^{-1} E\left(\tilde{D}^{-1}+Z^{k}\right)^{-1} E^{t} v^{k} \\
d f^{k} & =-\left(D_{f}^{k}\right)^{-1}\left(r_{a}^{k}-C^{t} d y^{k}\right) \\
d y(p)^{k} & =\left(\begin{array}{c}
-\left(D_{p}^{k}\right)^{-1} \\
0
\end{array}\right) d y^{k} \\
D_{p}^{k} & =\left(P^{k}\right)^{-1} Z_{p}^{k}+\left(S_{p}^{k}\right)^{-1} W_{p}^{k}+H \\
d p^{k} & =-\left(D_{p}^{k}\right)^{-1}\left(r_{b}^{k}+d y(p)^{k}\right) \\
d s_{f}^{k} & =r_{f}^{k}-d f^{k} \\
d s_{p}^{k} & =r_{2}^{k}-d p^{k} \\
d z_{f}^{k} & =\left(Z^{k}\right)^{-1}\left(r z f^{k}-Z_{f}^{k} d f^{k}\right) \\
d z_{p}^{k} & =\left(P^{k}\right)^{-1}\left(r_{z p}^{k}-Z_{p}^{k} d p^{k}\right) \\
d w_{f}^{k} & =\left(S_{f}^{k}\right)^{-1}\left(r_{w f}^{k}-W_{f}^{k} d s_{f}^{k}\right) \\
d w_{p}^{k} & =\left(S_{p}^{k}\right)^{-1}\left(r_{w p}^{k}-W_{p}^{k} d s_{p}^{k}\right) \\
d x^{k} & =\left(d f^{k}, d p^{k}, d s f^{k}, d s_{p}^{k}\right) \\
d t^{k} & =\left(d z_{f}^{k}, d w_{f}^{k}, d z_{p}^{k}, d w_{p}^{k}\right)
\end{aligned}
$$




$$
\begin{aligned}
\rho_{p}^{k} & =\frac{-1}{\min _{i}\left(\frac{d x^{k}}{x_{i}^{k}}\right)} \\
\rho_{d}^{k} & =\frac{-1}{\min _{i}\left(\frac{d t^{k}}{t_{i}^{k}}\right)} \\
\alpha_{p}^{k} & =\min \left(1, \tau \rho_{p}^{k}\right) \\
\alpha_{d}^{k} & =\min \left(1, \tau \rho_{d}^{k}\right) \\
\tilde{\gamma}^{k} & =\left(x^{k}+\alpha_{p}^{k} d x^{k}\right)^{t}\left(t^{k}+\alpha_{d}^{k} d t^{k}\right) \\
x^{k+1} & =x^{k}+\alpha^{k} d x^{k} \\
y^{k+1} & =y^{k}+\alpha^{k} d y^{k} \\
t^{k+1} & =t^{k}+\alpha^{k} d t^{k}
\end{aligned}
$$

Até convergir.

\subsubsection{Utilização de uma Árvore Geradora}

É possível permutar $\tilde{C}=\left[\begin{array}{ll}C & e_{j}\end{array}\right]$ obtendo a matriz

$$
\hat{C}=\left[\begin{array}{ccc}
G & e_{j} & N \\
T_{G} & 0 & T_{N}
\end{array}\right]
$$

onde $G$ forma uma árvore geradora da rede e $N$ contém os arcos adicionais. Além disso, podemos reordenar $\left[G e_{j}\right]$ de tal forma que esta matriz seja triangular. Eliminando, estas variáveis obtemos um sistema linear com a matriz $T_{N}-T_{G}\left[G e_{j}\right]^{-1} N$. Esta matriz tem a dimensão do número de laços independentes e pode ser decomposta antes de se iniciar o processo iterativo, pois só depende dos dados da rede.

\subsubsection{Formação da Matriz de Reatância}

A forma mais esparsa da matriz de reatância é aquela em que cada laço independente é o menor possível. Estes laços são denominados elementares. Esta matriz tem a vantagem adicional de que cada arco contribui no máximo duas vezes na formação de laços para redes planares. Assim, tomando-se os laços sempre na mesma orientação, obtem-se uma matriz do tipo incidência nó-arco. Nós não temos conhecimento de nenhum método para encontrar os laços elementares de uma rede [7]. Em [5] é apresentada uma heurística para este problema. 
Nesta seção, optou-se por estudar a rede de geração-transmissão como uma árvore geradora com arcos adicionais, pois esta formação leva a uma matriz de reatância cuja estrutura pode ser explorada com eficiência. Esta matriz contém um bloco diagonal representando os arcos adicionais da árvore geradora $\left(T_{N}\right)$ uma vez que estes arcos participam apenas em um único laço. Portanto, podemos eliminar $T_{N}$ em (4.11) obtendo a matriz $\left(\left[\begin{array}{ll}G & e_{j}\end{array}\right]-N T_{N}^{-1} T_{G}\right)$ - um sistema linear com a dimensão do número de barras que multiplicado por $[G e]^{-1}$ resulta em:

$$
I+\operatorname{sign}\left(T_{G}^{t}\right) T_{G}
$$

uma matriz simétrica em estrutura e definida positiva havendo métodos eficientes para sua decomposição [4].

Outra vantagem deste procedimento é que a matriz $T_{N}-T_{G}\left[\begin{array}{ll}G & e_{j}\end{array}\right]^{-1} N$ também torna-se definida positiva e simétrica em estrutura:

$$
T_{N}+T_{G} \operatorname{sign}\left(T_{G}^{t}\right)
$$

Este esquema pode ser utilizado para qualquer árvore geradora. Entretanto, a esparsidade da matriz de reatância depende da árvore utilizada. Para obter uma matriz esparsa a árvore geradora utilizada nesta seção é construída escolhendo a barra com maior grau como a raiz e todos seus vizinhos como filhos. A barra remanescente com maior grau é então acrescentada a árvore. Este procedimento é repetido até que todas as barras façam parte da árvore. O objetivo desta heurística é obter uma árvore com profundidade pequena de forma que os laços formados pelas linhas não pertencentes à árvore contenham poucas barras.

\subsubsection{Inicialização, Parâmetros e Critério de Convergência}

Para a implementação do método de pontos interiores utilizaremos:

- $\tau=0.99995$;

- $\sigma=\frac{1}{\sqrt{n}}$.

O ponto inicial adotado foi: 
- $f^{0}=s_{f}^{0}=\frac{p^{\max }}{2}$;

- $p^{0}=s_{p}{ }^{0}=\frac{p^{\max }}{2}$;

- $y^{0}=0$

- $z_{f}^{0}=w_{f}^{0}=(R+I) e$

- $z_{p}^{0}=w_{p}^{0}=e$.

O critério de convergência é dado por:

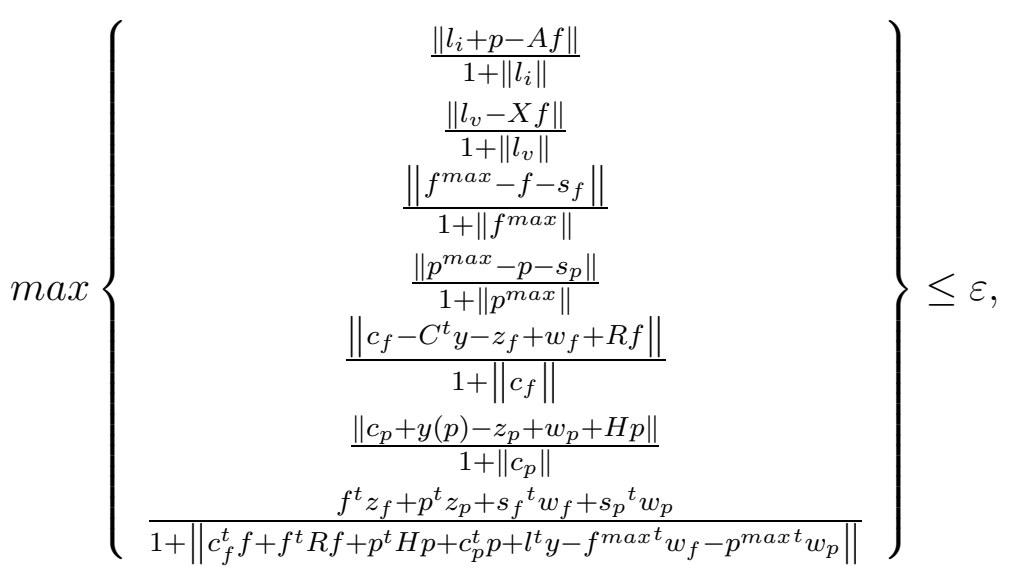

onde $\varepsilon$ é a tolerância estabelecida.

4.2 Métodos de Pontos Interiores Aplicados ao Fluxo de Potência Ótimo Usando o Princípio do Mínimo Esforço

\subsubsection{Método de Pontos Interiores Primal-Dual}

Considere o problema, descrito na seção (2.3):

$$
\begin{array}{cc}
\text { min } & \theta^{t} \tilde{B} \theta+p^{t} H p+c^{t} p \\
s a & B \theta-p=-l \\
& p^{\text {min }} \leq p \leq p^{\text {max }} .
\end{array}
$$


Fazendo a mudança de variáveis $\tilde{p}=p-p^{\text {min }}$ em (4.14), para simplificar o desenvolvimento do método, temos:

$$
\begin{array}{cc}
\min & \theta^{t} \tilde{B} \theta+\tilde{p}^{t} H \tilde{p}+c_{p}^{t} \tilde{p} \\
s a & B \theta-\tilde{p}=\tilde{l} \\
& 0 \leq \tilde{p} \leq \tilde{p}^{\text {max }}
\end{array}
$$

onde $c_{p}=2 H p^{\min }, \tilde{l}=p^{\min }-l$ e $\tilde{p}^{\max }=p^{\max }-p^{\min }$.

Adicionando a variável de folga e elimimando os "tils" de $p$ e $l$ (a fim de simplificar a notação) obtemos a formulação:

$$
\begin{array}{cc}
\min & \theta^{t} \tilde{B} \theta+p^{t} H p+c_{p}^{t} p \\
s a & B \theta-p=l \\
& p+s_{p}=p^{\max } \\
& \left(p, s_{p}\right) \geq 0 .
\end{array}
$$

Associado ao problema primal (4.15) temos o seguinte problema dual já com as variáveis de folga introduzidas:

$$
\begin{array}{cc}
\max & l^{t} y-\theta^{t} \tilde{B} \theta-\left(p^{\max }\right)^{t} w_{p}-p^{t} H p \\
s a & B^{t} y-\tilde{B} \theta=0 \\
& -y(p)+z_{p}-w_{p}-H p=c_{p} \\
& \left(z_{p}, w_{p}\right) \geq 0 .
\end{array}
$$

De posse das formulações primal e dual do problema iremos obter as condições de otimalidade que são dadas por:

factibilidade primal: $\left\{\begin{array}{c}B \theta-p=l \\ p+s_{p}=p^{\max } \\ \left(p, s_{p}\right) \geq 0\end{array}\right.$

factibilidade dual: $\left\{\begin{array}{c}B^{t} y-\tilde{B} \theta=0 \\ -y(p)+z_{p}-w_{p}-H p=c_{p} \\ \left(z_{p}, w_{p}\right) \geq 0\end{array}\right.$

condições de complementaridade: $\left\{\begin{array}{c}P Z_{p} e=0 \\ S_{p} W_{p} e=0 .\end{array}\right.$ 
Como vimos, o método de pontos interiores primal-dual pode ser desenvolvido através da aplicação do método de Newton às condições de otimalidade desconsiderando as restrições de não-negatividade e incluindo uma perturbação $(\mu)$ nas condições de complementaridade. O método parte de um ponto estritamente positivo e não permite que as variáveis se tornem negativas.

Então, aplicando o método de Newton, temos as seguintes direções:

$$
\left[\begin{array}{cccccc}
B & -I & 0 & 0 & 0 & 0 \\
0 & I & I & 0 & 0 & 0 \\
-\tilde{B} & 0 & 0 & B^{t} & 0 & 0 \\
0 & -H & 0 & -I & I & -I \\
0 & Z_{p} & 0 & 0 & P & 0 \\
0 & 0 & W_{p} & 0 & 0 & S_{p}
\end{array}\right]\left[\begin{array}{c}
d \theta \\
d p \\
d s_{p} \\
d y \\
d z_{p} \\
d w_{p}
\end{array}\right]=\left[\begin{array}{c}
r_{1} \\
r_{2} \\
r_{3} \\
r_{4} \\
r_{5} \\
r_{6}
\end{array}\right]
$$

Ou seja,

$$
\left\{\begin{array}{c}
B d \theta-d p=r_{1} \\
d p+d s_{p}=r_{2} \\
B^{t} d y-\tilde{B} d \theta=r_{3} \\
-d y(p)+d z_{p}-d w_{p}-H d p=r_{4} \\
Z_{p} d p+P d z_{p}=r_{5} \\
W_{p} d s_{p}+S_{p} d w_{p}=r_{6} .
\end{array}\right.
$$

Onde os resíduos são dados por:

$$
\left\{\begin{array}{c}
r_{1}=l-B \theta+p \\
r_{2}=p^{\max }-p-s_{p} \\
r_{3}=-B^{t} y+\tilde{B} \theta \\
r_{4}=c_{p}+y(p)-z_{p}+w_{p}+H p \\
r_{5}=\mu e-P Z_{p} e \\
r_{6}=\mu e-S_{p} W_{p} e
\end{array}\right.
$$

O sistema linear (4.17) pode ser simplificado através da eliminação de variáveis sem modificar sua estrutura esparsa. 
Substituindo as variáveis de folga, temos:

$$
\begin{aligned}
d w_{p} & =S_{p}^{-1}\left(r_{6}-W_{p} d s_{p}\right) \\
d z_{p} & =P^{-1}\left(r_{5}-Z_{p} d_{p}\right) \\
d s_{p} & =r_{2}-d p
\end{aligned}
$$

Com essas substituições, o sistema (4.17) se reduz a:

$$
\left\{\begin{array}{c}
B d \theta-d p=r_{1} \\
B^{t} d y-\tilde{B} d \theta=r_{3} \\
-d y(p)-D_{p} d p=r_{p}
\end{array}\right.
$$

onde $D_{p}=P^{-1} Z_{p}+H+S_{p}^{-1} W_{p}$ e $r_{p}=r_{4}-P^{-1} r_{5}+S_{p}^{-1} r_{6}-S_{p}^{-1} W_{p} r_{2}$.

Eliminando $d p$ da última equação de (4.18), temos:

$$
d p=-D_{p}^{-1}\left(r_{p}+d y(p)\right)
$$

Substituindo, então, no Sistema (4.18), temos:

$$
\left\{\begin{array}{c}
B d \theta+D_{p}^{-1}\left(r_{p}+d y(p)\right)=r_{1} \\
B^{t} d y-\tilde{B} d \theta=r_{3} .
\end{array}\right.
$$

Seja $D$ a matriz diagonal cujos elementos não nulos correspondem às barras de geração dados por $D_{p}^{-1}$. Logo:

$$
\left\{\begin{aligned}
B d \theta+D d y & =r_{a} \\
B^{t} d y-\tilde{B} d \theta & =r_{3}
\end{aligned}\right.
$$

onde $r_{a}=r_{1}-D r_{p}$.

Lembremos que $B$ e $\tilde{B}$ são matrizes quadradas, não singulares e que podem ser decompostas uma única vez antes do processo iterativo.

Podemos, portanto, eliminar $d \theta$ da segunda equação de (4.20), obtendo:

$$
d \theta=\tilde{B}^{-1}\left(B^{t} d y-r_{3}\right)
$$

e, substituindo na outra equação, temos:

$$
\begin{gathered}
B\left(\tilde{B}^{-1}\left(B^{t} d y-r_{3}\right)\right)+D d y=r_{a} \Longrightarrow \\
\left(B \tilde{B}^{-1} B^{t}+D\right) d y=r
\end{gathered}
$$


onde $r=r_{a}+B \tilde{B}^{-1} r_{3}$.

Utilizaremos novamente a fórmula de Sherman-Morrison-Woodbury [6] para obter $d y:$

$$
\begin{aligned}
W & =B^{-1} E \\
Z & =W^{t} \tilde{B} W \\
v & =\left(B \tilde{B}^{-1} B^{t}\right)^{-1} r=\left(B^{-t} \tilde{B} B^{-1}\right) r \\
d y & =v-\left(B \tilde{B}^{-1} B^{t}\right)^{-1} E\left(D^{-1}+Z\right)^{-1} E^{t} v
\end{aligned}
$$

onde $E$ é formada por vetores canônicos correspondentes aos elementos diagonais não nulos de $D$. $W$ e $Z$ são fixas para uma determinada rede e podem ser calculadas antes da aplicação do método iterativo.

Podemos, enfim, resumir o método de pontos interiores primal-dual:

Dados $\left(p^{0}, s_{p}^{0}, z_{p}^{0}, w_{p}^{0}\right) \geq 0, \theta^{0}$ e $y^{0}$ livres e $\sigma \in(0,1)$.

Sejam $x=\left(p, s_{p}\right), t=\left(z_{p}, w_{p}\right), n_{p}$ a dimensão do vetor $x$ e $\gamma^{k}$ o gap.

Para $k=0,1,2, \ldots$, faça:

$\mu^{k}=\sigma \frac{\gamma^{k}}{n_{p}}$.

Calcule os resíduos $r_{1}^{k}$ a $r_{6}^{k}$ e $r_{p}^{k}$.

$$
\begin{aligned}
D_{p}^{k} & =\left(P^{k}\right)^{-1} Z_{p}^{k}+H+\left(S_{p}^{k}\right)^{-1} W_{p}^{k} \\
r_{a}^{k} & =r_{1}^{k}-D^{k} r_{p}^{k} \\
r^{k} & =r_{a}^{k}+B \tilde{B}^{-1} r_{3}^{k} \\
v^{k} & =\left(B^{-t} \tilde{B} B^{-1}\right) r^{k} \\
d y^{k} & =v^{k}-\left(B^{-t} \tilde{B} B^{-1}\right) E\left(D^{-1}+Z\right)^{-1} E^{t} v^{k} \\
d \theta^{k} & =\tilde{B}^{-1}\left(B^{t} d y^{k}-r_{3}^{k}\right) \\
d p^{k} & =-\left(D_{p}^{k}\right)^{-1}\left(r_{p}^{k}+d y(p)^{k}\right) \\
d s_{p}^{k} & =r_{2}^{k}-d p^{k} \\
d z_{p}^{k} & =\left(P^{k}\right)^{-1}\left(r_{5}^{k}-Z_{p}^{k} d_{p}^{k}\right) \\
d w_{p}^{k} & =\left(S_{p}^{k}\right)^{-1}\left(r_{6}^{k}-W_{p}^{k} d s_{p}^{k}\right) \\
d x^{k} & =\left(d p^{k}, d s_{p}^{k}\right)
\end{aligned}
$$




$$
\begin{aligned}
d t^{k} & =\left(d z_{p}^{k}, d w_{p}^{k}\right) \\
\rho_{p}^{k} & =\frac{-1}{\min _{i}\left(\frac{d x^{k}}{x_{i}^{k}}\right)} \\
\rho_{d}^{k} & =\frac{-1}{\min _{i}\left(\frac{d t^{k}}{t_{i}^{k}}\right)} \\
\alpha_{p}^{k} & =\min \left(1, \tau \rho_{p}^{k}\right) \\
\alpha_{d}^{k} & =\min \left(1, \tau \rho_{d}^{k}\right) \\
\alpha^{k} & =\min \left(\alpha_{p}, \alpha_{d}\right) \\
\theta^{k+1} & =\theta^{k}+\alpha^{k} d \theta^{k} \\
x^{k+1} & =x^{k}+\alpha^{k} d x^{k} \\
y^{k+1} & =y^{k}+\alpha^{k} d y^{k} \\
t^{k+1} & =t^{k}+\alpha^{k} d t^{k}
\end{aligned}
$$

Até convergir.

Podemos destacar que a resolução de sistemas lineares envolve a maior parte do esforço computacional do método. Mas, visto que as matrizes $B$ e $\tilde{B}$ não variam a cada iteração, apenas uma decomposição destas matrizes é necessária. Podemos verificar também que $\left(D^{-1}+Z\right)^{-1}$ pode ser calculada com pouco esforço computacional, uma vez que sua dimensão é dada pelo número de geradores menos um.

\subsubsection{Desenvolvimento Alternativo do Método}

Como foi mostrado no Capítulo 2, Seção 2.3, para transformarmos a matriz $B$ numa matriz não singular, eliminamos a $i$-ésima linha e coluna de $B$, adotando assim a barra correspondente como referência angular $\left(\theta_{i}=0\right)$. Mas, para garantirmos que a linha e a coluna eliminadas correspondam a $\theta_{i}=0$, eliminamos uma linha e respectiva coluna correspondente a um gerador.

Esta técnica pode não ser eficiente quando utilizada para sistemas muito carregados, onde a eliminação de um gerador pode comprometer a resolução do problema. 
Uma idéia para solucionar este problema é eliminarmos apenas uma linha de $B$, e trabalharmos com a matriz não quadrada $B_{m-1 \mathrm{X} m}$.

Desta forma, podemos repetir o desenvolvimento do método de pontos interiores como na Seção 4.2.1, até obtermos o sistema linear

$$
\left\{\begin{aligned}
B d \theta+D d y & =r_{a} \\
B^{t} d y-\tilde{B} d \theta & =r_{3} .
\end{aligned}\right.
$$

Podemos escrever a matriz $B$ da seguinte forma:

$$
B=\left(\begin{array}{ll}
B^{\prime} & b
\end{array}\right),
$$

onde $B^{\prime}$ é uma matriz quadrada, não singular, de ordem $m-1$ e $b$ é um vetor coluna de ordem $m-1$.

Assim,

$$
B d \theta=\left(\begin{array}{ll}
B^{\prime} & b
\end{array}\right)\left(\begin{array}{c}
d \theta^{\prime} \\
d \theta_{m}
\end{array}\right)
$$

E, então, da primeira equação de (4.22), temos

$$
\begin{gathered}
B d \theta+D d y=r_{a} \Longrightarrow\left(\begin{array}{ll}
B^{\prime} & b
\end{array}\right)\left(\begin{array}{c}
d \theta^{\prime} \\
d \theta_{m}
\end{array}\right)+D d y=r_{a} \Longrightarrow \\
B^{\prime} d \theta^{\prime}+\left(\begin{array}{ll}
D & b
\end{array}\right)\left(\begin{array}{c}
d y \\
d \theta_{m}
\end{array}\right)=r_{a} \Longrightarrow B^{\prime} d \theta^{\prime}=r_{a}-\left(\begin{array}{ll}
D & b
\end{array}\right)\left(\begin{array}{c}
d y \\
d \theta_{m}
\end{array}\right) \Longrightarrow \\
d \theta^{\prime}=\left(B^{\prime}\right)^{-1}\left[\begin{array}{cc}
\left.r_{a}-\left(\begin{array}{ll}
D & b
\end{array}\right)\left(\begin{array}{c}
d y \\
d \theta_{m}
\end{array}\right)\right]
\end{array}\right.
\end{gathered}
$$

Podemos, também, escrever $\tilde{B}$ da seguinte forma:

$$
\tilde{B}=\left(\begin{array}{ll}
\tilde{B}^{\prime} & \tilde{b}
\end{array}\right)
$$

onde $\tilde{B}^{\prime}$ é uma matriz de ordem $m \times m-1$ e $\tilde{b}$ é um vetor coluna de ordem $m$.

Da segunda equação do sistema (4.2.1), temos

$$
B^{t} d y-\tilde{B} d \theta=r_{3} \Longrightarrow B^{t} d y-\tilde{B}^{\prime}\left(\begin{array}{c}
d \theta^{\prime} \\
d \theta_{m}
\end{array}\right)=r_{3} \Longrightarrow
$$




$$
\begin{gathered}
B^{t} d y-\tilde{B}^{\prime}\left(B^{\prime}\right)^{-1}\left[r_{a}-\left(\begin{array}{ll}
D & b
\end{array}\right)\left(\begin{array}{c}
d y \\
d \theta_{m}
\end{array}\right)\right]-\tilde{B}\left(\begin{array}{c}
0 \\
d \theta_{m}
\end{array}\right)=r_{3} \Longrightarrow \\
B^{t} d y+\tilde{B}^{\prime}\left(B^{\prime}\right)^{-1}\left(\begin{array}{ll}
D & b
\end{array}\right)\left(\begin{array}{c}
d y \\
d \theta_{m}
\end{array}\right)-\tilde{B}\left(\begin{array}{c}
0 \\
d \theta_{m}
\end{array}\right)=r_{3}+\tilde{B}^{\prime}\left(B^{\prime}\right)^{-1} r_{a} .
\end{gathered}
$$

Fazendo $r^{\prime}=r_{3}+\tilde{B}^{\prime}\left(B^{\prime}\right)^{-1} r_{a}$, temos:

$$
\begin{aligned}
& {\left[B^{t}+\tilde{B}^{\prime}\left(B^{\prime}\right)^{-1} D\right] d y+\left[\tilde{B}^{\prime}\left(B^{\prime}\right)^{-1} b-\tilde{b}\right] d \theta_{m}=r^{\prime} \Longrightarrow} \\
& \left(B^{t}+\tilde{B}^{\prime}\left(B^{\prime}\right)^{-1} D \quad \tilde{B}^{\prime}\left(B^{\prime}\right)^{-1} b-\tilde{b}\right)\left(\begin{array}{c}
d y \\
d \theta_{m}
\end{array}\right)=r^{\prime} .
\end{aligned}
$$

Resolvendo o sistema linear acima obtemos as novas direções do método de pontos interiores.

Na engenharia, costuma-se inserir uma restrição adicional com balanço de potência ou então retirar uma coluna de $B$.

\subsubsection{Método de Pontos Interiores Preditor-Corretor}

Sabemos que no método preditor-corretor dois sistemas lineares determinam as direções. Primeiramente, é calculada a direção afim, resolvendo o sistema linear abaixo:

$$
\left\{\begin{array}{c}
B d \tilde{\theta}-d \tilde{p}=r_{1} \\
d \tilde{p}+d \tilde{s_{p}}=r_{2} \\
B^{t} d \tilde{y}-\tilde{B} d \tilde{\theta}=r_{3} \\
-d y(p)+d \tilde{z_{p}}-d \tilde{w}_{p}-H d \tilde{p}=r_{4} \\
Z_{p} d \tilde{p}+P d \tilde{z_{p}}=\tilde{r_{5}} \\
W_{p} d \tilde{s_{p}}+S_{p} d \tilde{w}_{p}=\tilde{r_{6}} .
\end{array}\right.
$$

Onde os resíduos são dados por:

$$
\left\{\begin{array}{c}
r_{1}=l-B \theta+p \\
r_{2}=p^{\max }-p-s_{p} \\
r_{3}=-B^{t} y+\tilde{B} \theta \\
r_{4}=c_{p}+y(p)-z_{p}+w_{p}+H p \\
\tilde{r_{5}}=-P Z_{p} e \\
\tilde{r_{6}}=-S_{p} W_{p} e
\end{array}\right.
$$


Em seguida, a direção desejada é obtida resolvendo o seguinte sistema linear:

$$
\left\{\begin{array}{c}
B d \theta-d p=r_{1} \\
d p+d s_{p}=r_{2} \\
B^{t} d y-\tilde{B} d \theta=r_{3} \\
-d y(p)+d z_{p}-d w_{p}-H d p=r_{4} \\
Z_{p} d p+P d z_{p}=r_{5} \\
W_{p} d s_{p}+S_{p} d w_{p}=r_{6} .
\end{array}\right.
$$

Onde os novos resíduos são dados por:

$$
\left\{\begin{array}{c}
r_{5}=\mu e-P Z_{p} e-\Delta \tilde{P} \Delta \tilde{Z}_{p} \\
r_{6}=\mu e-S_{p} W_{p} e-\Delta \tilde{S}_{p} \Delta \tilde{W}_{p}
\end{array}\right.
$$

Podemos, enfim, resumir o método de pontos interiores preditor-corretor:

$\operatorname{Dados}\left(p^{0}, s_{p}^{0}, z_{p}^{0}, w_{p}^{0}\right) \geq 0, \theta^{0}$ e $y^{0}$ livres e $\tau \in(0,1)$.

Sejam $x=\left(p, s_{p}\right), t=\left(z_{p}, w_{p}\right)$ e $n_{p}$ a dimensão do vetor $x$.

Para $k=0,1,2, \ldots$, faça:

Calcule os resíduos $r_{1}^{k}$ a $r_{4}^{k},{\tilde{r_{5}}}^{k},{\tilde{r_{6}}}^{k}$ e $r_{p}^{k}$.

$$
\begin{aligned}
D_{p}^{k} & =\left(P^{k}\right)^{-1} Z_{p}^{k}+H+\left(S_{p}^{k}\right)^{-1} W_{p}^{k} \\
r_{a}^{k} & =r_{1}^{k}+D^{k} r_{p}^{k} \\
r^{k} & =r_{a}^{k}+B \tilde{B}^{-1} r_{3}^{k} \\
v^{k} & =\left(B^{-t} \tilde{B} B^{-1}\right) r^{k} \\
d \tilde{y}^{k} & =v^{k}-\left(B^{-t} \tilde{B} B^{-1}\right) E\left(D^{-1}+Z\right)^{-1} E^{t} v^{k} \\
d \tilde{\theta}^{k} & =\tilde{B}^{-1}\left(B^{t} d \tilde{y}^{k}-r_{3}^{k}\right) \\
d \tilde{p}^{k} & =-\left(D_{p}^{k}\right)^{-1}\left(r_{p}^{k}+d y(p)^{k}\right) \\
d \tilde{s}_{p}^{k} & =r_{2}^{k}-d \tilde{p}^{k} \\
d \tilde{z}_{p}^{k} & =\left(P^{k}\right)^{-1}\left(\tilde{r}_{5}^{k}-Z_{p}^{k} d \tilde{p}^{k}\right) \\
d \tilde{w}_{p}^{k} & =\left(S_{p}^{k}\right)^{-1}\left({\tilde{r_{6}}}^{k}-W_{p}^{k} d{\tilde{s_{p}}}^{k}\right) \\
d \tilde{x}^{k} & =\left(d \tilde{p}^{k}, d{\tilde{s_{p}}}^{k}\right) \\
d \tilde{t}^{k} & =\left(d \tilde{z}_{p}^{k}, d{\tilde{w_{p}}}^{k}\right)
\end{aligned}
$$




$$
\begin{aligned}
{\tilde{\rho_{p}}}^{k} & =\frac{-1}{\min _{i}\left(\frac{d \tilde{x}^{k}}{x_{i}^{k}}\right)} \\
{\tilde{\rho_{d}}}^{k} & =\frac{-1}{\min _{i}\left(\frac{d \tilde{t}^{k}}{t_{i}^{k}}\right)} \\
{\tilde{\alpha_{p}}}^{k} & =\min \left(1, \tau{\tilde{\rho_{p}}}^{k}\right) \\
{\tilde{\alpha_{d}}}^{k} & =\min \left(1, \tau{\tilde{\rho_{d}}}^{k}\right) \\
\tilde{\gamma}^{k} & =\left(x^{k}+{\tilde{\alpha_{p}}}^{k} d \tilde{x}^{k}\right)^{t}\left(t^{k}+{\tilde{\alpha_{d}}}^{k} d \tilde{t}^{k}\right)
\end{aligned}
$$

Calcule $\sigma^{k}$ e $\mu^{k}$.

Calcule os resíduos $r_{5}^{k}, r_{6}^{k}$ e $r_{s p}^{k}$.

$$
\begin{aligned}
& r_{a}^{k}=r_{1}^{k}+D^{k} r_{s p}^{k} \\
& r^{k}=r_{a}^{k}+B \tilde{B}^{-1} r_{3}^{k} \\
& v^{k}=\left(B^{-t} \tilde{B} B^{-1}\right) r^{k} \\
& d y^{k}=v^{k}-\left(B^{-t} \tilde{B} B^{-1}\right) E\left(D^{-1}+Z\right)^{-1} E^{t} v^{k} \\
& d \theta^{k}=\tilde{B}^{-1}\left(B^{t} d y^{k}-r_{3}^{k}\right) \\
& d p^{k}=-\left(D_{p}^{k}\right)^{-1}\left(r_{p}^{k}+d y(p)^{k}\right) \\
& d s_{p}^{k}=r_{2}^{k}-d p^{k} \\
& d z_{p}^{k}=\left(P^{k}\right)^{-1}\left(r_{5}^{k}-Z_{p}^{k} d p^{k}\right) \\
& d w_{p}^{k}=\left(S_{p}^{k}\right)^{-1}\left(r_{6}^{k}-W_{p}^{k} d s_{p}^{k}\right) \\
& d x^{k}=\left(d p^{k}, d s_{p}^{k}\right) \\
& d t^{k}=\left(d z_{p}^{k}, d w_{p}^{k}\right) \\
& \rho_{p}^{k}=\frac{-1}{\min _{i}\left(\frac{d x^{k}}{x_{i}^{k}}\right)} \\
& \rho_{d}^{k}=\frac{-1}{\min _{i}\left(\frac{d t^{k}}{t_{i}^{k}}\right)} \\
& \alpha_{p}^{k}=\min \left(1, \tau \rho_{p}^{k}\right) \\
& \alpha_{d}^{k}=\min \left(1, \tau \rho_{d}^{k}\right) \\
& \alpha^{k}=\min \left(\alpha_{p}, \alpha_{d}\right) \\
& \theta^{k+1}=\theta^{k}+\alpha^{k} d \theta^{k} \\
& x^{k+1}=x^{k}+\alpha^{k} d x^{k}
\end{aligned}
$$


$y^{k+1}=y^{k}+\alpha^{k} d y^{k}$

$t^{k+1}=t^{k}+\alpha^{k} d t^{k}$

Até convergir.

\subsubsection{Inicialização, Parâmetros e Critério de Convergência}

Para a implementação do método de pontos interiores utilizaremos:

- $\tau=0.99995$;

- $\sigma=\frac{1}{\sqrt{n}}$.

O ponto inicial adotado foi:

- $\theta^{0}=0$

- $p^{0}=s_{p}^{0}=\frac{p^{\max }}{2}$

- $y^{0}=0$;

- $z_{p}^{0}=w_{p}^{0}=e$.

O critério de convergência é dado por:

$$
\max \left\{\begin{array}{c}
\frac{\|l-B \theta+p\|}{1+\|l\|} \\
\frac{\left\|p^{\text {max }}-p-s_{p}\right\|}{1+\left\|p^{\text {max }}\right\|} \\
\frac{\left\|c_{p}+y(p)-z_{p}+w_{p}+H p\right\|}{1+\left\|c_{p}\right\|} \\
\frac{p^{t} z_{p}+s_{p}^{t} w_{p}}{1+\left\|\theta^{t} \tilde{B} \theta+p^{t} H p+c_{p}^{t} p+l^{t} y-p^{\text {maxt }} w_{p}\right\|}
\end{array}\right\} \leq \varepsilon .
$$




\section{Capítulo 5}

\section{Resultados Numéricos}

Mostraremos, neste capítulo, os resultados obtidos pela aplicação dos métodos de pontos interiores primal-dual e preditor-corretor ao problema de fluxo de potência ótimo e fluxo de potência ótimo usando o princípio do mínimo esforço.

A implementação dos métodos de pontos interiores para os problemas específicos foi feita em MATLAB 5.3.

\subsection{Sistemas Testes}

Utilizamos nesta implementação os sistemas testes que estão caracterizados abaixo:

1. IEEE30: representa um equivalente do sistema de energia elétrica do meio-oeste americano em dezembro de 1961.

2. IEEE118: representa um equivalente do sistema de energia elétrica do meio-oeste americano em dezembro de 1962.

3. SSC810: representa um equivalente do sistema de energia elétrica do sul/sudeste/centrooeste brasileiro.

4. SSC1654: representa um equivalente do sistema de energia elétrica do sul/sudeste/centrooeste brasileiro.

5. SSC1732: representa um equivalente do sistema de energia elétrica do sul/sudeste/centrooeste brasileiro. 
6. BRASIL1993: representa um equivalente do sistema interconectado brasileiro.

Cabe observar que o IEEE30 original tem dois geradores, mas ele foi modificado em nossos testes para conter seis geradores, seguindo uma tendência adotada em vários trabalhos.

As Figuras (5.1) e (5.2) representam os sistemas IEEE30 e IEEE118, respectivamente.

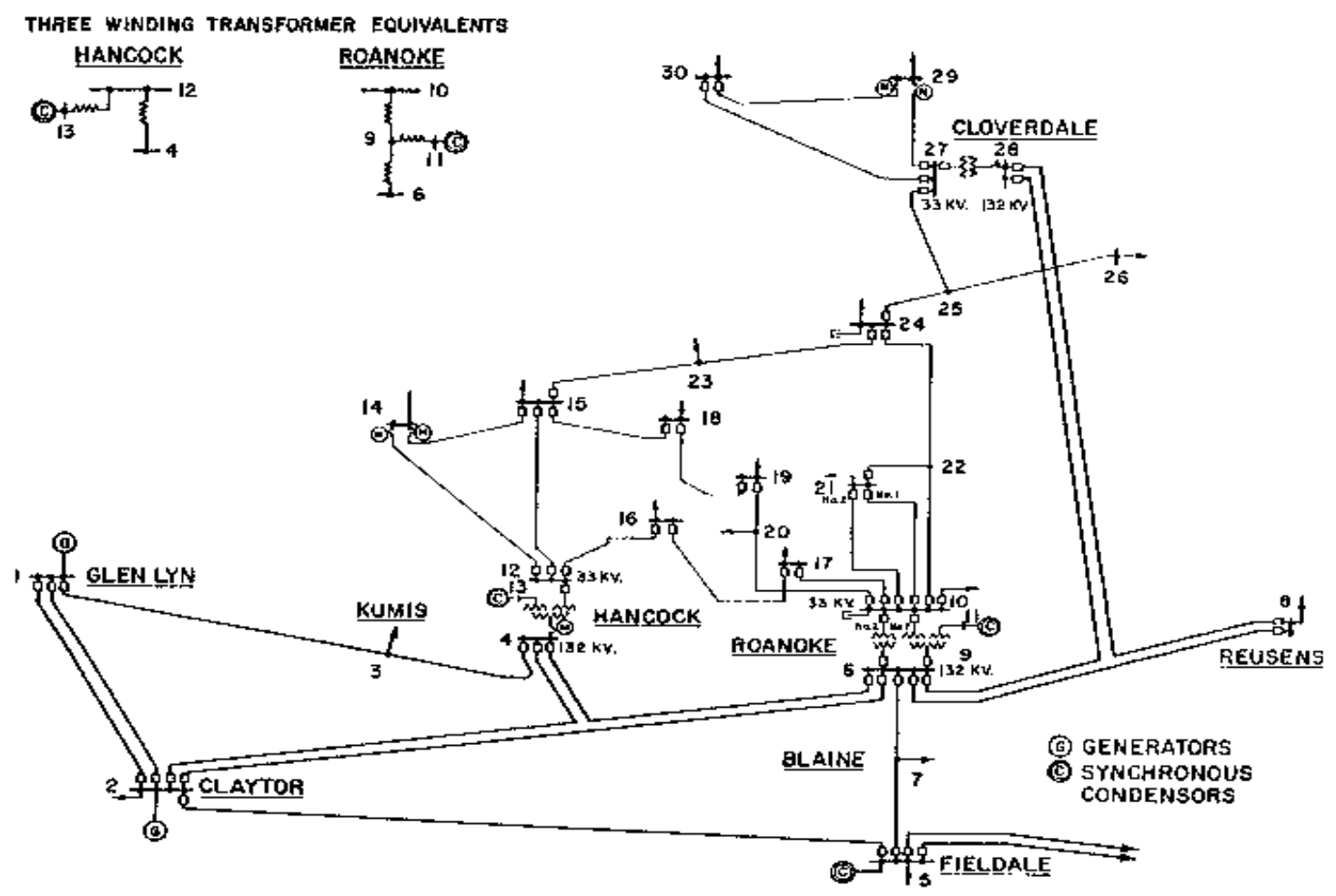

Figura 5.1: Mapa físico associado ao sistema teste IEEE30

\subsection{Resultados para o Sistema IEEE30}

Nesta seção, o sistema teste utilizado foi IEEE30. Os dados de carga deste sistema encontram-se na Tabela 5.1, e a Figura 5.3 contém uma representação gráfica do mesmo.

Para encontrarmos o valor da geração e fluxo de potência ótimos associados a um dado problema, que minimize as perdas de geração e transmissão, utilizamos os modelos 


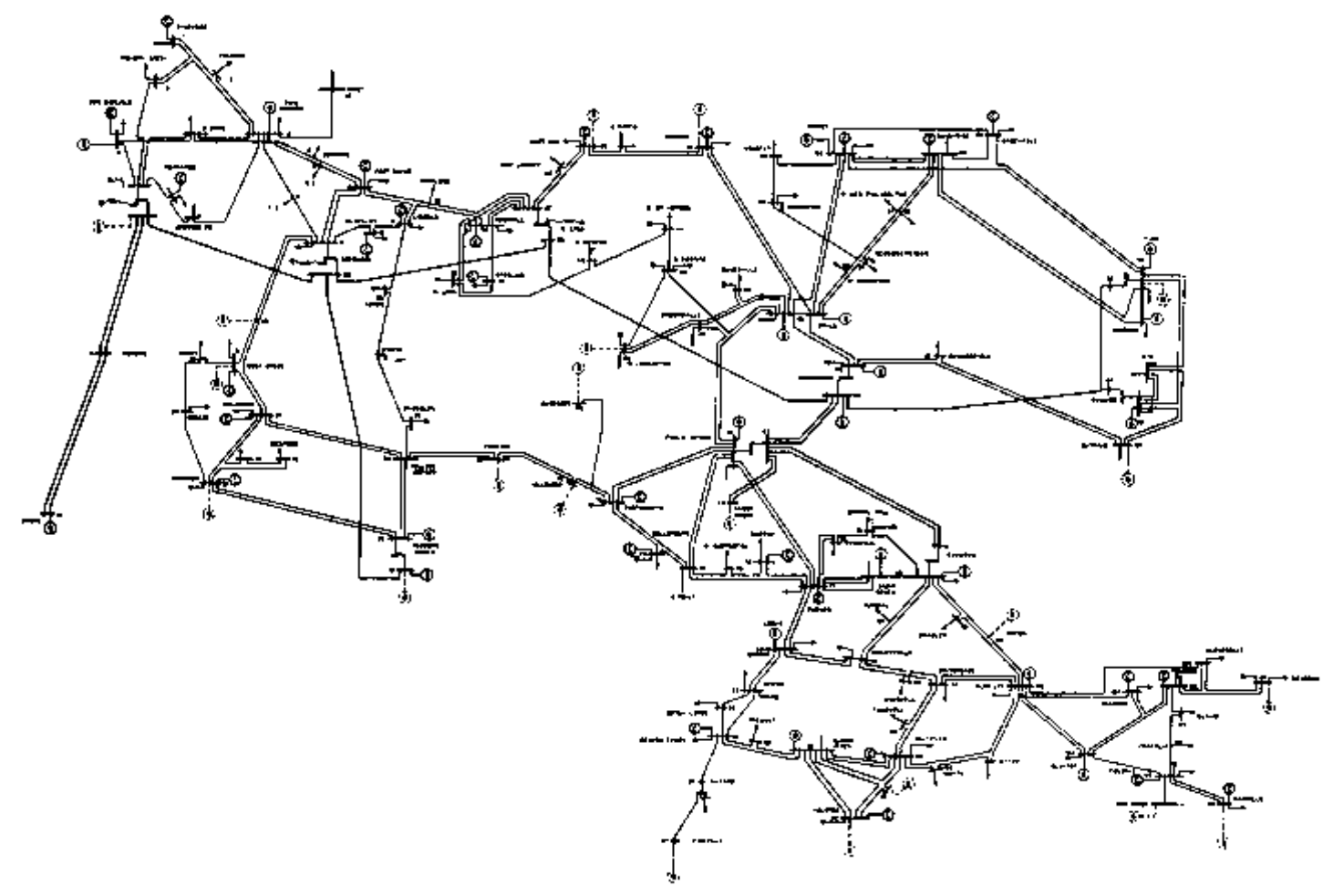

Figura 5.2: Mapa físico associado ao sistema teste IEEE118

matemáticos descritos no Capítulo 3. Os resultados obtidos fornecem o quanto deve ser gerado de energia e onde, bem como o valor do fluxo de potência para cada linha de transmissão.

Nestes experimentos foi adotado o valor $p^{\text {min }}=0$ para os geradores. Para simplificar a interpretação dos resultados, somente funções quadráticas puras foram utilizadas, ou seja, $c_{p}=0$, e os coeficientes quadráticos são os mesmos para todos os geradores exceto o de número cinco, que é duas vezes mais caro, e o de número um, que é duas vezes mais barato. O critério de parada utilizado é como o descrito na Seção 4.2.4 e escolhemos o valor de tolerância $\varepsilon=10^{-5}$.

Os limites de geração foram escolhidos de tal forma que na otimalidade não existam restrições de capacidade ativa. O despacho de geração é apresentado em três situações distintas, para cada problema, em termos das ponderações $\alpha$ e $\beta$ da função objetivo:

- T: Dá maior importância às perdas na transmissão( $\alpha=1$ e $\beta=0,1)$; 


\begin{tabular}{|c|c||c|c||c|c|}
\hline Barra & Carga & Barra & Carga & Barra & Carga \\
\hline 1 & 0,0 & 11 & 0,0 & 21 & 17,5 \\
\hline 2 & 21,7 & 12 & 11,2 & 22 & 0,0 \\
\hline 3 & 2,4 & 13 & 0,0 & 23 & 3,2 \\
\hline 4 & 7,6 & 14 & 6,2 & 24 & 8,7 \\
\hline 5 & 94,2 & 15 & 8,2 & 25 & 0,0 \\
\hline 6 & 0,0 & 16 & 3,5 & 26 & 3,5 \\
\hline 7 & 22,8 & 17 & 9,0 & 27 & 0,0 \\
\hline 8 & 30,0 & 18 & 3,2 & 28 & 0,0 \\
\hline 9 & 0,0 & 19 & 9,5 & 29 & 2,4 \\
\hline 10 & 5,8 & 20 & 2,2 & 30 & 10,6 \\
\hline
\end{tabular}

Tabela 5.1: IEEE30-Carga das barras em MW.

- G: Dá maior importância ao custo de geração $(\alpha=0,1$ e $\beta=1)$;

- G e T: Dá igual importância à geração e transmissão $(\alpha=\beta=1)$.

O tempo computacional, o número de iterações e de flops(número de operações de ponto flutuante) para cada uma das situações acima encontram-se nas Tabelas 5.2 e 5.3 para os problemas de fluxo de potência ótimo utilizando o modelo de fluxos em rede(MFR) e o princípio do mínimo esforço(PME), respectivamente.

\begin{tabular}{|c|c|c|c|c|}
\hline & Tempo(s) & Iterações & Flops & Flops Médio \\
\hline $\mathrm{T}$ & 0,01 & 5 & 37788 & 7558 \\
\hline $\mathrm{G}$ & 0,01 & 5 & 37802 & 7560 \\
\hline G e T & 0,01 & 5 & 37790 & 7558 \\
\hline
\end{tabular}

Tabela 5.2: Valores do desempenho do método para MFR.

Para melhor ilustrar os resultados obtidos em cada caso (T, G, G e T), para cada um dos problemas (MFR, PME) temos a Tabelas 5.4 e 5.5, e as Figuras 5.4 e 5.5, referentes à geração no IEEE30, em cada um dos testes realizados. 


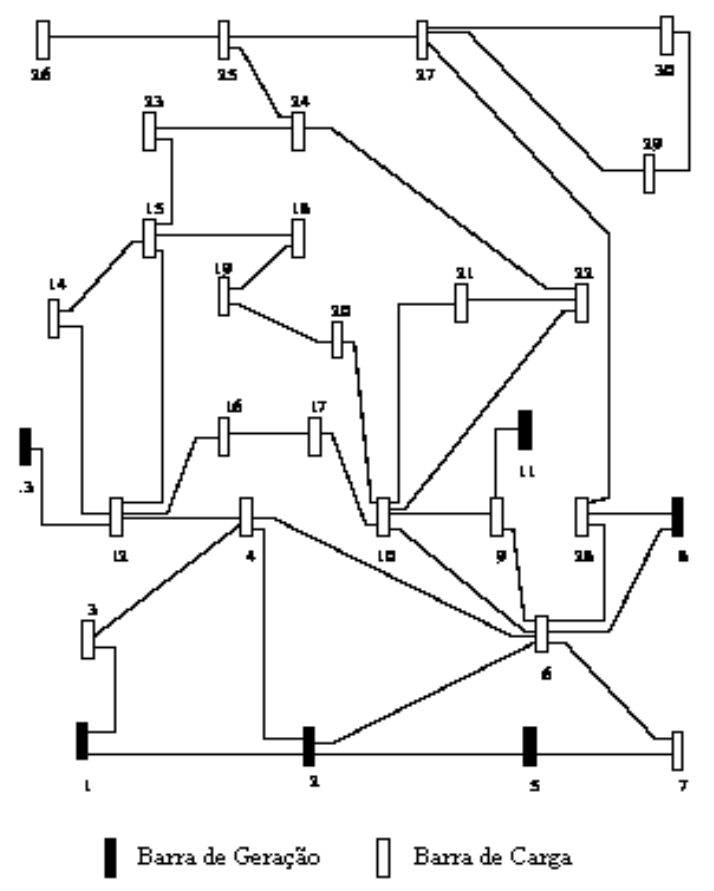

Figura 5.3: Sistema IEEE30

\begin{tabular}{|c|c|c|c|c|}
\hline & Tempo(s) & Iterações & Flops & Flops Médio \\
\hline $\mathrm{T}$ & 0,01 & 5 & 34081 & 6816 \\
\hline $\mathrm{G}$ & 0,01 & 5 & 34083 & 6817 \\
\hline G e T & 0,01 & 4 & 27233 & 6808 \\
\hline
\end{tabular}

Tabela 5.3: Valores do desempenho do método para PME.

Podemos verificar que os resultados obtidos pelos métodos para MFR e PME, em relação a geração de potência ativa, estão próximos, validando as implementações, uma vez que temos funções objetivo distintas para os dois problemas. Essa diferença na potência pode ser devida ao fato de que ignoramos as restrições de capacidade de transmissão, e podemos então estar violando algumas destas restrições.

As soluções obtidas considerando somente um dos objetivos a minimizar são muito diferentes entre si uma vez que o gerador mais barato (1) está muito mais distante da maior concentração de carga que o gerador mais caro(5). 


\begin{tabular}{|c|c|c|c|c|}
\hline Gerador & $\begin{array}{c}\text { Limite de } \\
\text { Geração }\end{array}$ & $\begin{array}{c}\text { Potência } \\
\text { Gerada(T) }\end{array}$ & $\begin{array}{c}\text { Potência } \\
\text { Gerada(G) }\end{array}$ & $\begin{array}{c}\text { Potência } \\
\text { Gerada(G e T) }\end{array}$ \\
\hline 1 & 100 & 5,91 & 67,83 & 23,62 \\
\hline 2 & 100 & 30,20 & 42,65 & 38,78 \\
\hline 5 & 100 & 98,86 & 32,99 & 71,48 \\
\hline 8 & 100 & 47,47 & 47,97 & 51,33 \\
\hline 11 & 100 & 68,60 & 49,56 & 62,22 \\
\hline 13 & 100 & 32,36 & 42,40 & 35,97 \\
\hline
\end{tabular}

Tabela 5.4: Valores de geração de potência ativa, em MW, para MFR.

\begin{tabular}{|c|c|c|c|c|}
\hline Gerador & $\begin{array}{c}\text { Limite de } \\
\text { Geração }\end{array}$ & $\begin{array}{c}\text { Potência } \\
\text { Gerada(T) }\end{array}$ & $\begin{array}{c}\text { Potência } \\
\text { Gerada(G) }\end{array}$ & $\begin{array}{c}\text { Potência } \\
\text { Gerada(G e T) }\end{array}$ \\
\hline 1 & 100 & 4,16 & 60,09 & 21,10 \\
\hline 2 & 100 & 26,81 & 38,05 & 35,98 \\
\hline 5 & 100 & 97,42 & 30,50 & 69,97 \\
\hline 8 & 100 & 35,35 & 40,69 & 42,64 \\
\hline 11 & 100 & 70,71 & 44,72 & 61,16 \\
\hline 13 & 100 & 35,44 & 39,49 & 37,36 \\
\hline
\end{tabular}

Tabela 5.5: Valores de geração de potência ativa, em MW, para PME.

\subsection{Resultados para o Sistema IEEE118}

Nesta seção, foram realizados diferentes testes utilizando o sistema IEEE118. Cada caso, corresponde a uma maneira de solucionar o sistema linear final de cada método (MFR e PME). Temos os seguintes casos:

- Caso 1: Vamos considerar aqui o MFR, com a utilização do comando interno do MATLAB para resolver o sistema linear (4.8).

- Caso 2: Consideraremos ainda o MFR, mas com a utlilização da fórmula de ShermanMorrison-Woodburry, como descrito na Seção 4.1.1, para a resolução do sistema linear (4.8), utlizando a estrutura (4.13) da Seção 4.1.4. 


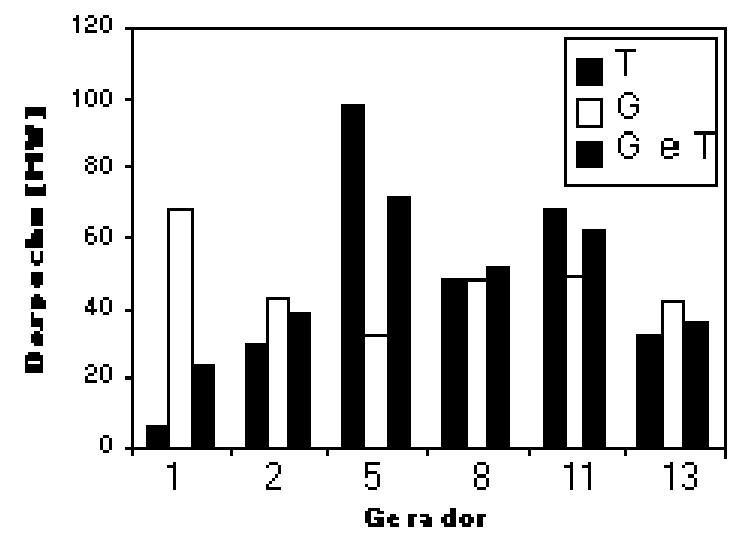

Figura 5.4: Despacho de potência ativa para ponderações distintas para MFR

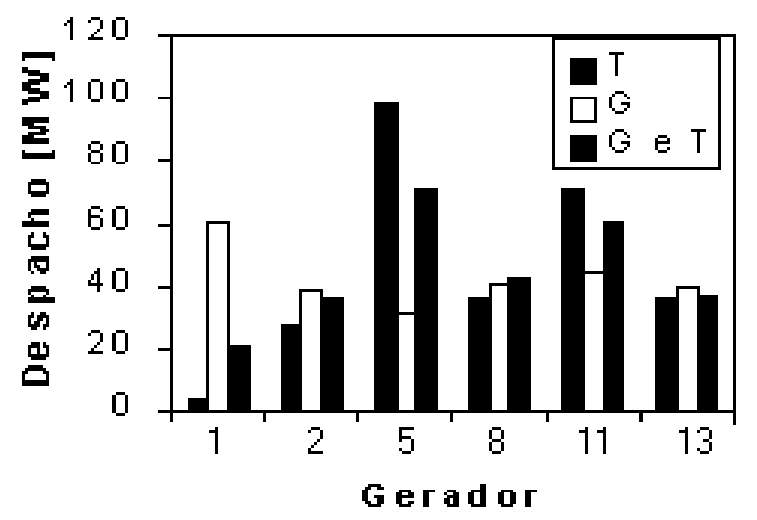

Figura 5.5: Despacho de potência ativa para ponderações distintas para PME

- Caso 3: Consideraremos o MFR, com a utlilização da fórmula de Sherman-MorrisonWoodburry, como descrito na Seção 4.1.1, para a resolução do sistema linear (4.8), utilizando a estrutura (4.12) da Seção 4.1.4.

- Caso 4: Vamos considerar o PME, com a utilização do comando interno do MATLAB para resolver o sistema linear (4.23).

- Caso 5: Consideraremos ainda o PME, com a utlilização da fórmula de ShermanMorrison-Woodburry, como descrito na Seção 4.2.1, para a resolução do sistema linear (4.23). 
Para melhor ilustrar os resultados obtidos em cada caso temos a Tabela 5.6, indicando o tempo computacional, o número de flops e de iterações para cada um dos testes realizados.

\begin{tabular}{|c|c|c|c|}
\hline & Tempo(s) & Iterações & Flops \\
\hline Caso 1 & 0,12 & 10 & 428058 \\
\hline Caso 2 & 0,19 & 10 & 9938197 \\
\hline Caso 3 & 0,19 & 10 & 10450717 \\
\hline Caso 4 & 0,13 & 6 & 6762865 \\
\hline Caso 5 & 0,06 & 6 & 600599 \\
\hline
\end{tabular}

Tabela 5.6: Resultados obtidos para o sistema IEEE118.

Observando a tabela, podemos dizer que, considerando o MFR, o Caso 1 se mostrou bem mais barato que os Casos 2 e 3 . Pudemos constatar que a grande desvantagem dos Casos 2 e 3 foi o cálculo da matriz $Z=W^{t} \tilde{D}_{f} W$, pois a matriz $W$ é uma matriz cheia, e $Z$ é recalculada a cada iteração. Seria possível reduzir os flops deste cáculo quase à metade guardando todos os produtos $W_{k i} W_{k j}$, mas usando muita memória. E depois dividir à metade novamente calculando só a parte inferior (superior) da $Z$, que é simétrica. Mas isso deveria ser feito em outra linguagem (C ou FORTRAN). No Caso 1, pudemos observar que o esforço computacional foi pequeno, devido a esparsidade da matriz do sistema (4.8).

Já no caso do PME, podemos observar que o Caso 5 realizou menos operações que o Caso 4. O grande custo computacional do Caso 4 se deve ao fato de a matriz do sistema linear (4.23) ser praticamente densa, e a vantagem do Caso 5 é que não contamos o cáculo de $Z$, ou seja, $Z$ é calculada apenas uma vez, antes do processo iterativo, e durante o processo, somente é utilizada a decomposição desta matriz.

Baseados nos resultados obtidos nesta seção, vamos escolher como padrão para os próximos testes o melhor caso, para cada uma das abordagens (MFR e PME), ou seja, Caso 1 e Caso 5, respectivamente. 


\subsection{Resultados para os Demais Sistemas}

Para os sistemas testados aqui, não iremos exibir todos os valores de geração e transmissão obtidos devido a grande quantidade de dados.

Nestes experimentos também adotamos os valores $p^{\text {min }}=0$ e $c_{p}=0$. Os coeficientes quadráticos são os mesmos para todos os geradores.

Então para mostrar o desempenho do método, mostraremos o tempo computacional em segundos, o número de iterações, de flops e de flops médio (número de operações de ponto flutuante por iteração) para cada um dos sistemas.

Temos os resultados para o método primal-dual e preditor-corretor para os problemas de fluxo de potência ótimo(PD-MFR e PC-MFR, respectivamente), e fluxo de potência ótimo usando o princípio do mínimo esforço(PD-PME, PC-PME).

Para maior clareza do texto, vamos escrever uma tabela para cada problema testado contendo os resultados obtidos para o mesmo.

- Sistema IEEE30, com pmax = 50MW: Ver Tabela 5.7;

\begin{tabular}{|c|c|c|c|c|}
\hline & Tempo(s) & Iterações & Flops & Flops Médio \\
\hline PD-MFR & 0,03 & 7 & 52878 & 7554 \\
\hline PC-MFR & 0,02 & 6 & 69704 & 11617 \\
\hline PD-PME & 0,02 & 8 & 54630 & 6829 \\
\hline PC-PME & 0,01 & 6 & 68468 & 11411 \\
\hline
\end{tabular}

Tabela 5.7: Valores do desempenho dos métodos para IEEE30.

- Sistema IEEE118, com pmax = 100MW: Ver Tabela 5.8;

- Sistema SSC810, com pmax =4500MW: Ver Tabela 5.9;

- Sistema SSC1654, com pmax = 2500MW: Ver Tabela 5.10;

- Sistema SSC1732, com pmax = 3500MW: Ver Tabela 5.11; 


\begin{tabular}{|l|c|c|c|c|}
\hline & Tempo(s) & Iterações & Flops & Flops Médio \\
\hline PD-MFR & 0,16 & 10 & 428058 & 42806 \\
\hline PC-MFR & 0,15 & 7 & 470685 & 67241 \\
\hline PD-PME & 0,08 & 6 & 600599 & 100100 \\
\hline PC-PME & 0,09 & 4 & 541101 & 135275 \\
\hline
\end{tabular}

Tabela 5.8: Valores do desempenho dos métodos para IEEE118.

\begin{tabular}{|c|c|c|c|c|}
\hline & Tempo(s) & Iterações & Flops & Flops Médio \\
\hline PD-MFR & 3,31 & 6 & 3134946 & 522491 \\
\hline PC-MFR & 2,85 & 4 & 3773277 & 943319 \\
\hline PD-PME & 2,69 & 5 & 4214034 & 842807 \\
\hline PC-PME & 2,63 & 3 & 3301261 & 1100420 \\
\hline
\end{tabular}

Tabela 5.9: Valores do desempenho dos métodos para SSC810.

- Sistema BRASIL1993, com pmax = 5000MW: Ver Tabela 5.12.

A modelagem baseada no princípio do mínimo esforço é superior à por fluxo em redes com relação ao número de iterações. Apesar de realizar mais iterações, a abordagem por fluxo em redes é mais rápida para os problemas de maior dimensão, uma vez que suas iterações são mais baratas que as da abordagem baseada no princípio do mínimo esforço. Podemos verificar também que todas as abordagens são rápidas e robustas, convergindo para todos os problemas testados.

Os sistemas de menor dimensão requerem um número maior de iterações que os sistemas maiores, possivelmente devido aos sistemas menores estarem mais carregados. 


\begin{tabular}{|c|c|c|c|c|}
\hline & Tempo(s) & Iterações & Flops & Flops Médio \\
\hline PD-MFR & 11,99 & 7 & 7481382 & 1068769 \\
\hline PC-MFR & 10,80 & 5 & 9531362 & 1906272 \\
\hline PD-PME & 41,83 & 4 & 19072492 & 3814498 \\
\hline PC-PME & 41,35 & 2 & 16303972 & 8515986 \\
\hline
\end{tabular}

Tabela 5.10: Valores do desempenho dos métodos para SSC1654.

\begin{tabular}{|c|c|c|c|c|}
\hline & Tempo(s) & Iterações & Flops & Flops Médio \\
\hline PD-MFR & 13,10 & 7 & 7688240 & 1098320 \\
\hline PC-MFR & 11,67 & 5 & 9844461 & 1968892 \\
\hline PD-PME & 49,14 & 4 & 21038948 & 5259737 \\
\hline PC-PME & 48,71 & 2 & 18304309 & 9152155 \\
\hline
\end{tabular}

Tabela 5.11: Valores do desempenho dos métodos para SSC1732.

\begin{tabular}{|c|c|c|c|c|}
\hline & Tempo(s) & Iterações & Flops & Flops Médio \\
\hline PD-MFR & 13,31 & 6 & 5067732 & 844622 \\
\hline PC-MFR & 10,29 & 4 & 5782112 & 1445528 \\
\hline PD-PME & 29,01 & 4 & 13884960 & 3471240 \\
\hline PC-PME & 28,74 & 2 & 10686731 & 5343366 \\
\hline
\end{tabular}

Tabela 5.12: Valores do desempenho dos métodos para BRASIL. 


\section{Capítulo 6}

\section{Conclusões e Perspectivas Futuras}

Observando os resultados numéricos podemos concluir que:

- Não é vantajoso utilizar a fórmula de Sherman-Morrison-Woodburry para resolver o sistema linear final da abordagem MFR, pois temos que o cálculo da matriz $Z$ é muito caro, e tem de ser feito em todas iterações. O contrário ocorre para a abordagem PME, visto que não calculamos a matriz $Z$ durante o processo iterativo.

- Podemos concluir também que, no caso do PME, não é vantajosos utilizar o comando interno do MATLAB para resolver o sistema linear final, visto que a matriz deste sistema é praticamente densa. Já para o caso da MFR, isso é vantajoso, devido a esparsidade da matriz do sistema linear.

- Em ambas abordagens, o método de pontos interiores preditor-corretor obteve melhor desempenho que o primal-dual, conseguindo um tempo computacional menor, mesmo considerando maior esforço por iteração. Podemos ver que o esforço por iteração do método preditor-corretor realmente é menor que o dobro do esforço do primal-dual, uma vez que resolvemos dois sistemas lineares utilizando o mesmo Jacobiano. E, para a abordagem PME, podemos verificar que o método preditorcorretor obteve um custo computacional menor que o primal-dual.

- Podemos observar que os sistemas de menor dimensão são resolvidos em um número maior de iterações que os sistemas maiores. Uma possível explicação para isso é que sistemas menores estão mais carregados. 
- Os métodos de pontos interiores se mostraram robustos, convergindo bem mesmo para problemas bastante sobrecarregados, sem apresentar instabilidade numérica. Podemos verificar que todas as abordagens são rápidas, cabendo destacar que o maior número de iterações foi 10, mesmo para sistemas muito carregados.

- Podemos concluir também que a modelagem baseada no princípio do mínimo esforço é superior à por fluxo em redes em número de iterações. Mas, para problemas de maior dimensão, a modelagem por fluxo em redes se mostra mais rápida, mesmo realizando mais iterações.

Temos as seguintes possibilidades de trabalhos futuros:

- Fazer a implementação dos métodos em outras linguagens (C ou FORTRAN), a fim de tentar diminuir o esforço computacional, principalmente no cálculo da matriz $Z$, para o MFR.

- Fazer o estudo da estrutura matricial do sistema resultante do desenvolvimento do método primal-dual para o problema baseado no princípio do mínimo esforço, utilizando a nova idéia de modificação da matriz $B$, visando obter um método mais eficiente e mais barato.

- Implementar o método citado acima e comparar os resultados com os já obtidos pelos outros métodos.

- Verificar se há violação nas restrições de capacidade de transmissão para a modelagem baseada no princípio do mínimo esforço e, se houver utilizar o processo citado para resolvê-la. 


\section{Bibliografia}

[1] I. Adler, M. G. C. Resende, G. Veiga and N. Karmarkar, An Implementation of Karmarkar's Algorithm for Linear Programming, Mathematical Programming, 44, (1989), pp. 297-335.

[2] M. F. Carvalho, S. Soares and T. Ohishi, Optimal Active Power Dispatch by Network Flow Approach, IEEE Transactions on Power Systems, 3(3), (1988), pp. 1640-1647.

[3] J. Castro, A Specialized Interior-Point Algorithm for Multcommodity Network Flows , SIAM J. Optimization, 10(3), (2000), pp. 852-877.

[4] I. S. Duff, A. M. Erisman and J. K. Reid, Direct Methods for Sparse Matrices, Clarendon Press, Oxford, (1986).

[5] P. Franco, M. F. Carvalho and S. Soares, A Network Flow Model for Short-term Hydrodominated Hydrotermal Scheduling Problem , IEEE Transactions on Power Systems, 9(2), (1994), pp. 1016-1021.

[6] G. H. Golub, V. Loan and F. Charles , Matrix Computations Third Edition , The Johns Hopkins University Press, Baltimore, Maryland, (1996).

[7] M. Gondran and M. Minoux, "Graphs and algorithms", Chichester : J. Wiley, (1986).

[8] J. Gondzio, Multiple Centrality Corrections in a Primal-Dual Method for Linear Programming, Computational Optimization and Applications, 6, (1996), pp. 137-156.

[9] N. Karmarkar, A New Polynomial-Time Algorithm for Linear Programming, Combinatorica, 4(4), (1984), pp. 373-395. 
[10] J. L. Kennington and R. V. Helgason, Algorithms for Network Programming, Wiley, New York, (1980).

[11] I. J. Lustig, R. E. Marsten and D. F. Shanno, On Implementing Mehrotra's PredictorCorrector Interior Point Method for Linear Programming, SIAM J. Optimization, 2, (1992), pp. 435-449.

[12] S. Mehrotra, On the Implementation of a Primal-Dual Interior Point Method, SIAM Journal on Optimization, 2(4), (1992), pp. 575-601.

[13] J. A. Momoh, M. E. El-Hawary and R. Adapa, A Review of Selected Optimal Power Flow Literature to 1993,Part II Newton, Linear Programming and Interior Point Methods, IEEE Transactions on Power Systems, 14(1), (1999), pp. 105-111.

[14] R.D.C. Monteiro, I. Adler and M.G.C. Resende, A Polynomial-time Primal-Dual Affine Scaling Algorithm for Linear and Convex Quadratic Programming and its Power Series Extension ,Mathematics of Operations Research, 15, (1990), pp191-214.

[15] A. Monticelli, A. Santos, M. V. Pereira, S. H. Cunha, B. J. Parker and J. C. Praça, Interactive Transmission Network Planning Using a Least Effort Criterion, IEEE Transactions on Power Apparatus and Systems, 10, (1982), p. 101.

[16] A. A. Oliveira, Uma Metodologia para o Cálculo do Spot Price em Sistemas Hidrotérmicos , Tese de Mestrado, FEEC, UNICAMP, Campinas, SP, (1999).

[17] A.R.L. Oliveira and C. Lyra, Implementação de um Método de Pontos Interiores para Programação Linear, SBA: Controle \& Automação, 3(2), (1991), pp370-382.

[18] A. R. L. Oliveira and C. Lyra, Interior Point Methods for the Polynomial $L_{\infty}$ Fitting Problem, Internacional Transactions in Operational Research 11(3), (2004), pp309-322.

[19] A. R. L. Oliveira, M. A. Nascimento and C. Lyra, Efficient Implementation and Benchmark of Interior Point Methods for the Polynomial $L_{1}$ Fitting Problem, Statistics \& Data Analysis, 35, (2000), pp. 119-135. 
[20] A. R. L. Oliveira, L. Nepomuceno and S. Soares, Optimal Active Power Dispatch Combining Network Flow and Interior Point Approaches, IEEE Transactions on Power Systems, 18(4), (2003), pp.1235-1240.

[21] A. R. L. Oliveira, L. Nepomuceno and S. Soares, Short Term Hydroelectric Scheduling Combining Network Flow and Interior Point Approaches, Trabalho aceito pela Electrical Power \& Energy Systems, (2004).

[22] A. R. L. Oliveira and S. Soares, Métodos de Pontos Interiores para Problema de Fluxo de Potência Ótimo DC , SBA: Controle \& Automação, 14(3), (2003), pp.278-285.

[23] V. H. Quintana, G. L. Torres and J. Medina-Palomo, Interior Point Methods and their Applications to Power Systems: A Classification of Publications and Software Codes , IEEE Transactions on Power Systems, 15(1), (2000), pp.170-176.

[24] M. G. C. Resende and G. Veiga, An Efficient Implementation of a Network Interior Point Method, DIMACS Series in Discrete Mathematics and Theoretical Computer Science, 12, (1993), pp.299-348.

[25] S. Soares and C. T. Salmazo, Minimum Loss Predispatch Model for Hydroelectric Systems , IEEE Transactions on Power Systems, 12(3), (1997), pp.1220-1228.

[26] R. J. Vanderbei, Linear Programming - Foundations and Extensions , Kluwer Academics Publishers, Boston, USA, (1996).

[27] S. J. Wright, Primal-Dual Interior-Point Methods , SIAM Publications, SIAM, Philadelphia, PA, USA, (1996). 